\title{
The Gaia-ESO Survey: The inner disc, intermediate-age open cluster Pismis 18 ${ }^{\star}$
}

D. Hatzidimitriou ${ }^{1,2}$, E. V. Held ${ }^{3}$, E. Tognelli ${ }^{4,5}$, A. Bragaglia ${ }^{6}$, L. Magrini $^{7}$, L. Bravi $^{7}$, K. Gazeas $^{1}$, A. Dapergolas $^{2}$, A. Drazdauskas $^{8}$, E. Delgado-Mena ${ }^{9}$, E. D. Friel ${ }^{10}$, R. Minkevičiūtè ${ }^{8}$, R. Sordo ${ }^{3}$, G. Tautvaišiené ${ }^{8}$, G. Gilmore ${ }^{11}$, S. Randich ${ }^{7}$, S. Feltzing $^{12}$, A. Vallenari ${ }^{3}$, E. J. Alfaro ${ }^{13}$, E. Flaccomio ${ }^{14}$, A. C. Lanzafame $^{15}$, E. Pancino ${ }^{6}$, R. Smiljanic ${ }^{16}$, A. Bayo ${ }^{17}$, M. Bergemann ${ }^{18}$, G. Carraro ${ }^{19}$, A. R. Casey ${ }^{20,21}$, M. T. Costado ${ }^{22}$, F. Damiani ${ }^{14}$, E. Franciosini ${ }^{7}$, A. Gonneau ${ }^{11}$, P. Jofré ${ }^{23}$, J. Lewis ${ }^{11}$, L. Monaco ${ }^{24}$, L. Morbidelli ${ }^{6}$, C. C. Worley ${ }^{11}$, and S. Zaggia ${ }^{3}$

${ }^{1}$ Section of Astrophysics, Astronomy and Mechanics, Department of Physics, National and Kapodistrian University of Athens, 15784 Athens, Greece e-mail: dh@physics.uoc.gr

2 IAASARS, National Observatory of Athens, 15236 Penteli, Greece

3 INAF, Osservatorio Astronomico di Padova, Vicolo dell'Osservatorio 5, 35122 Padova, Italy

Dipartimento di Fisica "E.Fermi", Universitá di Pisa, Largo Bruno Pontecorvo 3, 56127 Pisa, Italy

5 INFN, Sezione di Pisa, Largo Bruno Pontecorvo 3, 56127 Pisa, Italy

6 INAF, Osservatorio di Astrofisica e Fisica dello Spazio di Bologna, Via Gobetti 93/3, 40129 Bologna, Italy

7 INAF, Osservatorio Astrofisico di Arcetri, Largo E. Fermi 5, 50125 Firenze, Italy

8 Institute of Theoretical Physics and Astronomy, Vilnius University, Sauletekio av. 3, 10257 Vilnius, Lithuania

9 Instituto de Astrofísica e Ciências do Espaço, Universidade do Porto, CAUP, Rua das Estrelas, 4150-762 Porto, Portugal

10 Astronomy Department, Indiana University Bloomington, Swain West 319, 727 East 3rd Street, Bloomington, IN 47405-7105, USA

11 Institute of Astronomy, Cambridge University, Madingley Road, Cambridge CB3 OHA, UK

12 Lund Observatory, Department of Astronomy and Theoretical Physics, Box 43, 22100 Lund, Sweden

13 Instituto de Astrofísica de Andalucía, Camino Bajo de Huétor, 50, 18008 Granada, Spain

14 INAF, Osservatorio Astronomico di Palermo, Piazza del Parlamento 1, 90134 Palermo, Italy

15 Dipartimento di Fisica e Astronomia, Università di Catania, Italy

16 Nicolaus Copernicus Astronomical Center, Polish Academy of Sciences, ul. Bartycka 18, 00-716 Warsaw, Poland

17 Instituto de Física y Astronomía, Fac. de Ciencias, Universidad de Valparaíso, Gran Bretana 1111, Playa Ancha, Chile

18 Max-Planck Institut für Astronomie, Königstuhl 17, 69117 Heidelberg, Germany

19 Dipartimento di Fisica e Astronomia Galileo Galilei, Universitá di Padova, Vicolo Osservatorio 3, 35122 Padova, Italy

20 School of Physics and Astronomy, Monash University, Clayton 3800, Victoria, Australia

21 Faculty of Information Technology, Monash University, Clayton 3800, Victoria, Australia

22 Departamento de Didáctica, Universidad de Cśdiz, 11519 Puerto Real, Cádiz, Spain

23 Núcleo de Astronomía, Facultad de Ingeniería y Ciencias, Universidad Diego Portales (UDP), Santiago, Chile

24 Departamento de Ciencias Fisicas, Universidad Andres Bello, Fernandez Concha 700, Las Condes, Santiago, Chile

Received 13 November 2018 / Accepted 14 February 2019

\section{ABSTRACT}

Context. Pismis 18 is a moderately populated, intermediate-age open cluster located within the solar circle at a Galactocentric distance of about seven kpc. Few open clusters have been studied in detail in the inner disc region before the Gaia-ESO Survey.

Aims. New data from the Gaia-ESO Survey allowed us to conduct an extended radial velocity membership study as well as spectroscopic metallicity and detailed chemical abundance measurements for this cluster.

Methods. Gaia-ESO Survey data for 142 potential members, lying on the upper main sequence and on the red clump, yielded radial velocity measurements, which, together with proper motion measurements from the Gaia Second Data Release (Gaia DR2), were used to determine the systemic velocity of the cluster and membership of individual stars. Photometry from Gaia DR2 was used to re-determine cluster parameters based on high confidence member stars only. Cluster abundance measurements of six radial-velocity member stars with UVES high-resolution spectroscopy are presented for 23 elements.

Results. The average radial velocity of 26 high confidence members is $-27.5 \pm 2.5(\mathrm{std}) \mathrm{km} \mathrm{s}^{-1}$ with an average proper motion of

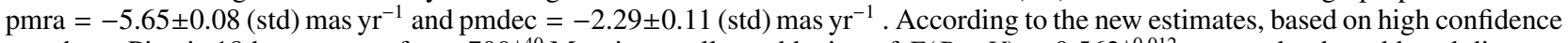
members, Pismis 18 has an age of $\tau=700_{-50}^{+40} \mathrm{Myr}$, interstellar reddening of $E(B-V)=0.562_{-0.026}^{+0.012}$ mag and a de-reddened distance modulus of $\mathrm{DM}_{0}=11.96_{-0.24}^{+0.10}$ mag. The median metallicity of the cluster (using the six UVES stars) is $[\mathrm{Fe} / \mathrm{H}]=+0.23 \pm 0.05$ dex, with $[\alpha / \mathrm{Fe}]=0.07 \pm 0.13$ and a slight enhancement of $s$ - and $r$-neutron-capture elements.

Conclusions. With the present work, we fully characterized the open cluster Pismis 18. We confirmed its present location in the inner disc. We estimated a younger age than the previous literature values and we gave, for the first time, its metallicity and its detailed abundances. Its $[\alpha / \mathrm{Fe}]$ and [ $s$-process/Fe], both slightly super-solar, are in agreement with other inner-disc open clusters observed by the Gaia-ESO survey.

Key words. stars: abundances - open clusters and associations: individual: Pismis 18 - Galaxy: abundances - Galaxy: formation Galaxy: disk

* Full Table 2 is only available at the CDS via anonymous ftp to cdsarc.u-strasbg.fr (130.79.128.5) or via http://cdsarc.u-strasbg.fr/viz-bin/qcat?J/A+A/626/A90 


\section{Introduction}

Open clusters (OCs), being simple populations with relatively easily determined ages, are among the best tracers of the chemical evolution of the Galactic thin disc from its outer regions to the Galactic bulge (e.g. Friel 1995; Sestito et al. 2008; Yong et al. 2012; Donati et al. 2015; Magrini et al. 2015; Cantat-Gaudin et al. 2016; Netopil et al. 2016; Casamiquela et al. 2017).

The inner disc $\left(R_{\mathrm{GC}}<8 \mathrm{kpc}\right)$ is an area of particular importance as it constitutes a link between the properties of the bulge and of the thin/thick disc. In order to probe the chemical evolution of the inner disc one needs information both on ages and on abundances of stars or stellar populations, which can be provided by a detailed study of chemical abundances of intermediate age and old clusters with known ages (see, e.g. Jacobson et al. 2016). Relatively old OCs are quite rare in these high density regions due to high mortality rates (Portegies Zwart et al. 1998; Kruijssen et al. 2011). Thus the percentage of OCs with ages larger than about one Gyr in the inner disc is only $\simeq 11 \%$, based on the catalogue of inner disc clusters by Morales et al. (2013). Moreover, inner disc clusters are often difficult to observe due to high field contamination and heavy and/or differential reddening.

Pismis 18 (IC 4291) is considered to be an intermediate age OC with an age of about one Gyr, located in the inner disc, at a distance of about $2.77 \mathrm{kpc}$ (according to a new determination by Cantat-Gaudin et al. (2018a) based on Gaia DR2 parallaxes and proper motions) from the Sun in the direction of the Galactic Centre. It lies within the fourth quadrant of the Galactic plane on the Sagittarius arm. In the study of Morales et al. (2013) it is classified as a totally exposed cluster with no correlation with sub-millimetre dust continuum emission.

Pismis 18 is included in the Gaia-ESO survey (GES), which is a large, public spectroscopic survey of the Galaxy carried out with the high-resolution multi-object spectrograph Fiber Large Array Multi-Element Spectrograph (FLAMES; Pasquini et al. 2002) on the Very Large Telescope (ESO, Chile). The aim of the survey is to provide accurate radial velocities and detailed element abundances for about $10^{5}$ stars covering the bulge, thick and thin discs, and halo components, as well as a sample of about 65 OCs of all ages, metallicities, locations, and masses (cf. Gilmore et al. 2012; Randich \& Gilmore 2013). Pismis 18 is the seventh inner disc open cluster individually studied within the framework of the GES, the other clusters being NGC 4815 (Friel et al. 2014), NGC 6705 (Cantat-Gaudin et al. 2014), Be 81 (Magrini et al. 2015), Tr 20 (Donati et al. 2014), Tr 23 (Overbeek et al. 2017) and NGC 6802 (Tang et al. 2017). Other studies have focused more on the general properties of the cluster population as a whole (see, e.g. Magrini et al. 2015, 2017; Spina et al. 2017; Bravi et al. 2018; Randich et al. 2018).

The purpose of this paper is to present our GES observations of Pismis 18, with the aim of providing a detailed membership analysis, abundances for 23 elements and revised cluster parameters (age, distance, metallicity and reddening) based on high confidence members. Previous studies of Pismis 18 are summarized in Sect. 2. In Sect. 3 we describe the target selection, observations and data analysis, while in Sect. 4 we perform the selection of high confidence members based on their proper motions (from the Gaia Second Data Release; hereafter, Gaia DR2, Gaia Collaboration 2018b) and GES radial velocities. Sect. 5 provides atmospheric parameters for the high confidence member stars. Based on Gaia DR2 photometry of the high confidence cluster members, along with the newly determined metal abundance of the cluster, a revised set of cluster parameters is determined in Sect. 6, and the element abundance measurements are discussed in Sect. 7. Our results are summarized in Sect. 8.

\section{Pismis 18 in the literature}

The only published optical photometry for Pismis 18 is the CCD $B V I$ photometry of Piatti et al. (1998), who also obtained an integrated spectrum for this cluster. Their colour-magnitude diagram (CMD) reveals a well-defined main sequence (MS) and red clump (RC) with relatively little contamination by field stars, partly due to the small field of view $\left(4 \times 4 \operatorname{arcmin}^{2}\right)$. They have estimated the age of Pismis 18 at $1.2 \pm 0.4 \mathrm{Gyr}$ (on the basis of the CMD and the integrated spectrum), with a reddening value of $E(B-V)=0.50 \pm 0.05$ and a distance of $2.24 \pm 0.41 \mathrm{kpc}$, assuming solar abundance. More recently, Tadross (2008) has analysed 2MASS data to construct $J H K$ CMDs. He has estimated an age of $0.8 \mathrm{Gyr}$ (using solar metallicity isochrones from Bonatto et al. 2004), interstellar reddening of $E(B-V)=0.61$ and a distance of $1790 \pm 82 \mathrm{pc}$. He has also provided revised values for the cluster central coordinates and extent, giving a diameter of 5.6 arcmin, larger than the one tabulated in Dias et al. (2002). The estimates provided by the two studies for the age, reddening and distance of the cluster are marginally consistent within the errors (see Sect. 6). Kharchenko et al. (2013) have redetermined the cluster parameters using 2MASS photometry, proper motions, and solar metallicity isochrones. There is no spectroscopic determination of the metallicity of Pismis 18 based on individual stars, although Piatti et al. (1998) obtained a rough estimate of the metallicity, $[\mathrm{Fe} / \mathrm{H}]=0.0$, from the width of CaII triplet lines in integrated spectra.

Pismis 18 is too distant to be included in the Gaia-TGAS catalogue (Gaia Collaboration 2017; Cantat-Gaudin et al. 2018b). However, as mentioned in the Introduction, it is featured in Gaia DR2 (see e.g. Cantat-Gaudin et al. 2018a) and we use the information in the present paper (relevant parameters from this study are shown in Col. 6 of Table 1). Table 1 summarizes the literature values of the Pismis 18 parameters, together with the ones derived in the present paper (described in Sect. 6).

Finally, 16 stars in Pismis 18 have been observed by Carlberg (2014) with the MIKE spectrograph at a resolution of $\sim 44000$. These spectra are part of a study of rotation in open clusters, so their $\mathrm{S} / \mathrm{N}$ is low and only permitted derivation of radial velocities (RV) and $v \sin i$. Twelve stars have been classified as members of the cluster with $\langle\mathrm{RV}\rangle=-27.9 \pm 0.8 \mathrm{~km} \mathrm{~s}^{-1}$. We have seven stars in common with this study, indicated in Col. 18 of Table 2.

\section{Observations and data reduction}

The target selection, observation, data reduction, atmospheric parameter determination and abundance measurements were handled within the GES collaboration by specific working groups (WGs). The targets in Pismis 18 were selected following the strategy applied for all intermediate-age OCs with prominent red clumps (RC) (see, e.g. Bragaglia 2018). Briefly, the RC stars, which have high cluster membership probability, were observed with UVES with the aim of determining precise chemical abundances. Stars on the MS were observed with GIRAFFE, using the HR9B setup primarily for stars of spectral type A to F and the HR15N setup for cooler stars (see below for more details). The selection of GIRAFFE targets was aimed at observing an inclusive and unbiased sample of cluster star candidates rather than only high probability members, with the purpose of defining cluster membership using the RVs obtained with the larger GIRAFFE sample. 
Table 1. Pismis 18 basic parameters.

\begin{tabular}{lcccccc}
\hline \hline Property & WEBDA & Piatti et al. (1998) & Tadross (2008) & Kharchenko et al. (2013) & Cantat-Gaudin et al. (2018a) & Present study \\
\hline RA (J2000) & $13: 36: 55$ & $13: 36: 32^{(a)}$ & $13: 36: 56$ & $13: 36: 55.8$ & $13: 36: 54.5$ & $13: 36: 58.1$ \\
Dec (J2000) & $-62: 05: 36$ & $-62: 12: 48^{(a)}$ & $-62: 05: 45$ & $-62: 03: 54$ & $-62: 05: 28$ & $-62: 05: 35$ \\
$D$ (kpc) & 2.24 & $2.24 \pm 0.41$ & $1.79 \pm 0.08$ & 2.3 & $2.77^{(b)}$ & $2.47_{-0.26}^{+0.11}$ \\
$R_{\mathrm{GC}}(\mathrm{kpc})$ & & - & 7.53 & - & 15.1 & $6.8 \pm 0.1^{(d)}$ \\
$z$ (pc) & & - & & - & $2.88^{(e)}$ & $13 \pm 2$ \\
Radius (arcmin) & & $1.2 \pm 0.4$ & 0.8 & 0.94 & \multicolumn{2}{c}{} \\
Age (Gyr) & & $0.50 \pm 0.05$ & 0.61 & 0.52 & $0.70_{-0.05}^{+0.04}$ \\
$E(B-V)$ & 0.50 & 0.0 & - & - & $0.562_{-0.026}^{+0.012}$ \\
{$[\mathrm{Fe} / \mathrm{H}]$} & & & & & $0.23 \pm 0.05 \mathrm{dex}$ \\
\hline
\end{tabular}

Notes. ${ }^{(a)}$ Converted from the B1950 coordinates given in the paper. ${ }^{(b)}$ Most likely distance. ${ }^{(c)}$ Distance from Galactic midplane. ${ }^{(d)}$ The adopted distance of the Sun to the Galactic Centre is eight kpc (see Malkin et al. 2013). ${ }^{(e)}$ Radius including $50 \%$ of the cluster stars.

The MS targets were selected from the MS turnoff down to $V \simeq 19$. The GES observations nicely complement with RV the precise astrometric information of the Gaia mission for stars not reached by the Gaia RVS instrument (Gaia DR2 has RVs only for stars brighter than $G=12$, Gaia Collaboration 2018d) and permit a complete chemical characterisation of the cluster with the giants observed with UVES.

The targets were selected on the basis of the VPHAS+ ESO survey data (Drew et al. 2014) in the $r$ and $i$-bands (Vega system), as VPHAS+ provides homogeneous spatial coverage over the entire extent of the cluster. In total, we observed ten stars in the $\mathrm{RC}$ region and 132 stars on the MS. The left panel of Fig. 1 displays the $r$ vs. $(r-i)$ CMD over a region of 15 arcmin around the centre of Pismis 18. We also mark the selected targets on the MS and the RC. On the right panel of Fig. 1, we show a similar diagram based on Gaia DR2 photometry. It must be emphasized that not all targets (selected on the basis of VPHAS+) have Gaia DR2 photometry in all three bandpasses. It is noted that on the diagram on the right the main sequence is somewhat narrower for fainter magnitudes, although a direct comparison is not possible due to the different filters used.

The data for Pismis 18 were obtained in May and June 2014 with FLAMES on the VLT-UT2 telescope at the European Southern Observatory. The ten candidate RC stars were observed with the high-resolution spectrograph Ultraviolet and Visual Echelle Spectrograph (UVES, Pasquini et al. 2002) using the U580 setup (4800-6800 $\AA$ and $R=47000$ ), with total exposure times of either $12 \mathrm{ks}$ or $15 \mathrm{ks}$.

Spectra were obtained with the medium-resolution multifibre spectrograph GIRAFFE for 51 MS stars with $13<V<16$ $(12.7<r<15.6)$ using the HR9B setup $(5143-5356 \AA$ and $R=25900)$, while 91 MS stars with $15.5<V<19(15.1<$ $r<18.5)$ were observed using the HR15N setup (6470-6790 and $R=17000)$. It is noted that ten stars were in common between the two configurations. The total exposure times were $15 \mathrm{ks}$ for the HR15N setup and $12 \mathrm{ks}$ for the HR9B setup. The median signal-to-noise ratio was 116, 74 and 52 for the UVES, GIRAFFE HR15N and GIRAFFE HR9B spectra, respectively.

Data reduction included sky subtraction, barycentric correction and normalisation, as well as calculation of radial and rotational velocities. For details on the data reduction pipeline, specifically for the UVES spectra, see Sacco et al. (2014).

Parameter and abundance determinations for each target were typically performed by multiple nodes (WG sub-groups) in charge of abundance analysis. The results of individual nodes were combined within each WG; the WG values were then homogenized by WG15 (Hourihane et al., in prep.) to yield final recommended parameters, using a set of calibrators to define a common scale (Pancino et al. 2017). This structure produced homogeneous parameter determinations while allowing WGs to specialize in different types of stars. More details can be found, for example, in Overbeek et al. (2017) and references therein. The data described here came from the fifth internal data release (GES iDR5) which comprises a re-analysis of all available spectra until December 2015 using an updated linelist (Heiter et al. 2015) and state-of-the-art analysis techniques.

In Table 2, available only at the CDS in its entirety, we provide for the 142 observed stars, the GES ID number (CNAME), the Gaia DR2 ID, the equatorial coordinates (J2000) in degrees, the setup used for the observations, the derived radial velocities, the Gaia DR2 magnitudes BP, $G$ and $\mathrm{R} p$, the gri magnitudes from VPHAS+, the 2MASS $J H K$ magnitudes, the WEBDA (a site devoted to Stellar Clusters in the Galaxy and the Magellanic Clouds, developed and maintained by Ernst Paunzen and Christian Stütz, Institute of Astronomy of the University of Vienna (Austria) ${ }^{1}$ ) identification number whenever available, the Piatti et al. (1998) BVI photometry, the angular distance from the cluster centre $\left(r_{\text {centre }}\right)$ (in arcmin), the radial velocity from Carlberg (2014) for the common stars, and the parallax (in mas) and proper motion (in mas $\mathrm{yr}^{-1}$ ) according to Gaia DR2. The label " $\mathrm{m}$ " indicates high confidence membership assigned according to the analysis described in the next section.

\section{Membership determination}

As field contamination is quite significant in OCs, membership determination using radial velocities and proper motions is very important for the derivation of high confidence values for the cluster parameters. Accurate proper motions are now available from Gaia DR2 (Gaia Collaboration 2016, 2018b).

\subsection{Proper motions}

The Gaia DR2 recently provided useful information for the assessment of cluster membership of the targets in our sample. A recent study by Cantat-Gaudin et al. (2018a) analysed the Gaia DR2 catalogues to derive the membership of stars in a large sample of open clusters, including Pismis 18. They employed the unsupervised membership assignment code UPMASK (Krone-Martins \& Moitinho 2014) to give a membership probability to each star from proper motions and parallaxes, by taking into account all errors and correlations

\footnotetext{
1 https://www . univie.ac.at/webda/
} 

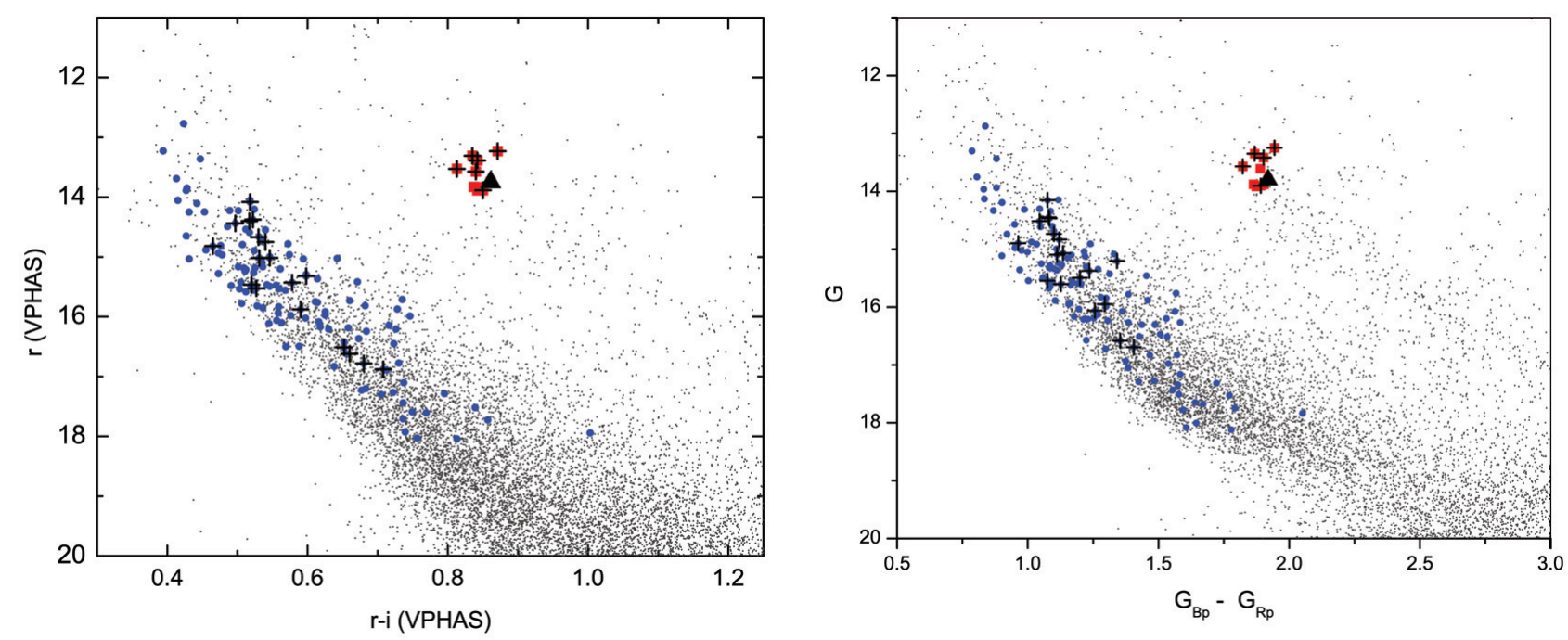

Fig. 1. Left panel: VPHAS+ colour magnitude diagram of Pismis 18 over the entire field of view of FLAMES, marked as small grey filled circles. The targets observed with UVES are marked with red filled squares, while targets observed with GIRAFFE are marked with blue filled circles. High confidence cluster members are indicated with black crosses and the possible binary star with a black filled triangle. Right panel: same, but for Gaia DR2 photometry.

between the parameters. We took advantage of this analysis to identify a set of probable cluster members among our observed stars. To this aim, our target list was matched with the Gaia DR2 catalogue using the 2MASS identifiers and the pre-computed crossmatch between Gaia DR2 and 2MASS ("gaiadr2.tmass_best_neighbour"). The latter was preferred as the result of a careful analysis by the Gaia team, including proper motion propagation and epoch correction (Marrese et al. 2019). Only stars with a 5-parameter solution were considered. We found 132 stars in common with Gaia DR2, whose proper motions are plotted in Fig. 2 (blue dots). Then, our catalogue was matched with the results of Cantat-Gaudin et al. (2018a) using the unique Gaia DR2 identifiers. We selected the spectroscopic targets with membership probability $P>0.5$ to define a sample of 35 probable members based on astrometry. These are plotted as red dots with error bars in Fig. 2. Using this sample we computed the average value and standard devia-

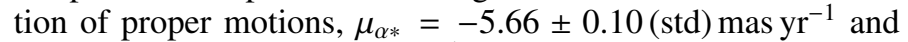
$\mu_{\delta}=-2.29 \pm 0.15$ (std) mas $\mathrm{yr}^{-1}$.

The selection of probable cluster members is refined in the next sub-section using the radial velocity information. The proper motions have not been corrected for the systematic uncer-

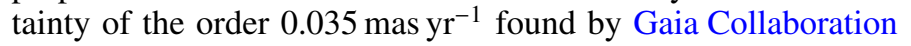
(2018c).

The Gaia DR2 also provides parallaxes for our 35 cluster member candidates. The mean parallax is quite well defined at $0.335 \pm 0.054$ (std) mas. However, we refrained from using this value to compute a geometric distance to Pismis 18 since there is evidence from previous studies that parallaxes in Gaia DR2 are affected by systematic errors of the order $0.03-0.05$ mas in the parallax absolute zero point (Lindegren et al. 2018; Luri et al. 2018). The distance to the cluster is discussed in Sect. 6 .

We mention here a special case represented by a UVES star (CNAME=13365001-6205376, marked with a square in Fig. 2), which has a proper motion close to that of the probable members, yet it is not present in the Cantat-Gaudin et al. (2018a) list. This star might possibly be an unresolved binary, in which case its parallax and proper motion as well as radial velocity could be incorrect. In fact, in Gaia DR2 all sources were treated as single stars in deriving the astrometric solution (Lindegren et al. 2018;

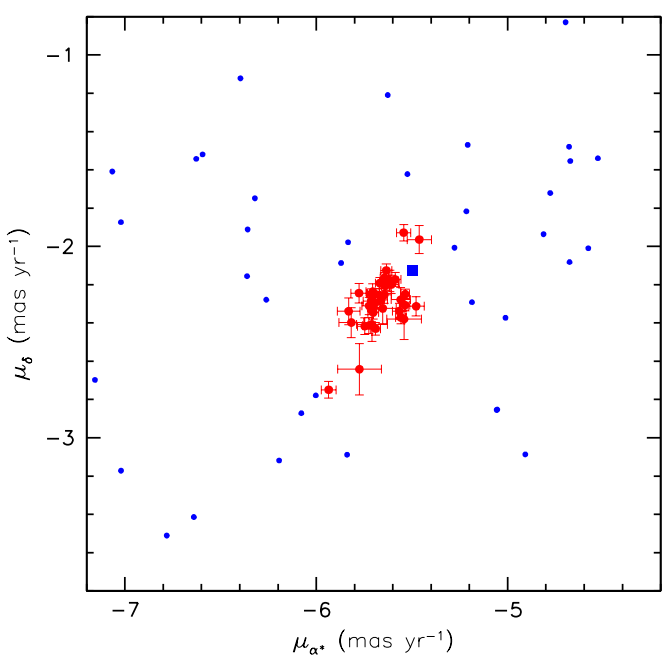

Fig. 2. Proper motions of stars in our Pismis 18 spectroscopic sample in common with the Gaia DR2. Red dots represent the probable members according to the analysis of Cantat-Gaudin et al. (2018a), with proper motion errors in the Gaia DR2. A blue square marks a suspect binary star observed with UVES (CNAME 13365001-6205376, see discussion in the text). Only a limited range of proper motions is shown here to highlight the locus of cluster member candidates.

Gaia Collaboration 2018b). Although not considered as a probable member in the following analysis, this star is included in our tables for future reference as a possible cluster member.

\subsection{Radial velocities}

The 35 proper motion likely members selected in the previous sub-section, were further analysed on the basis of their radial velocities, in order to construct the final catalogue of high confidence members based on both proper motions and radial velocities. The entire sample of the 142 observed Pismis 18 targets, given in Table 2, shows a wide range of radial velocities (from -63 to $+186 \mathrm{~km} \mathrm{~s}^{-1}$ ) with a broad peak around $-24 \mathrm{~km} \mathrm{~s}^{-1}$ with a standard deviation of $26 \mathrm{~km} \mathrm{~s}^{-1}$ (shown in grey in Fig. 3). 


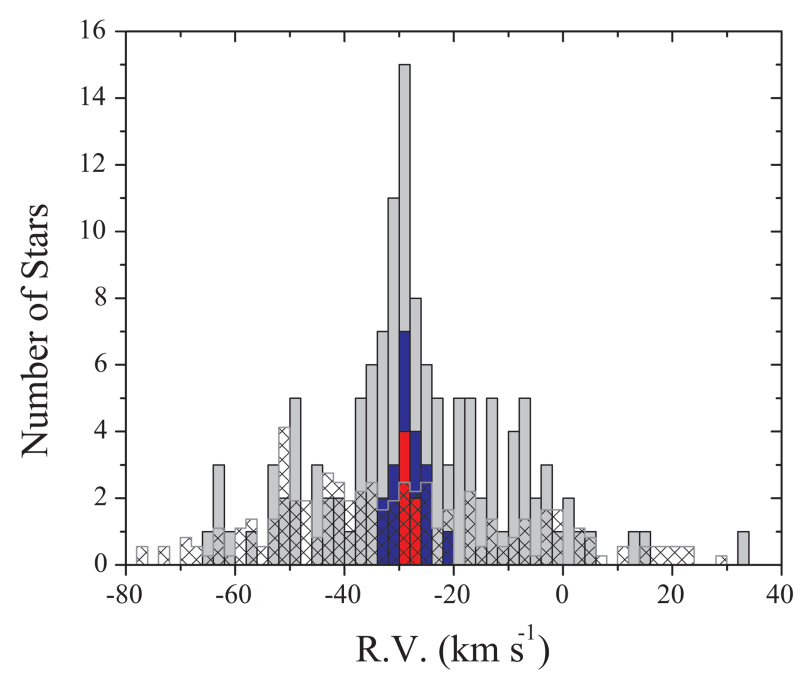

Fig. 3. Distribution of radial velocities of all 142 observed stars (in grey). The distribution of the GIRAFFE radial velocity members is shown in blue and that of the UVES radial velocity members in red. The patterned bars show the distribution of radial velocities of Galactic stars in the direction of Pismis 18 according to the Besançon star count model for the Galaxy. For the normalization of this curve see text.

The 35 proper motion likely members have a much tighter radial velocity distribution (from -61.7 to $-13.9 \mathrm{~km} \mathrm{~s}^{-1}$ ), with an average of -28.0 and a standard deviation of $7.4 \mathrm{~km} \mathrm{~s}^{-1}$. It must be noted that the radial velocity errors for individual stars are quite low, specifically, the median error was $\sim 0.51 \mathrm{~km} \mathrm{~s}^{-1}$ for HR $15 \mathrm{~N}$, $0.54 \mathrm{~km} \mathrm{~s}^{-1}$ for HR9B and $0.36 \mathrm{~km} \mathrm{~s}^{-1}$ for UVES (these values refer to the entire sample of observed targets). Stars with high rotational velocities or low signal-to-noise ratios can have significantly higher errors (several $\mathrm{km} \mathrm{s}^{-1}$, cf. Jackson et al. 2015). We have thus excluded from further analysis stars with radial velocity errors larger than $5 \mathrm{~km} \mathrm{~s}^{-1}$.

For the remaining 32 proper motion likely members with radial velocity errors less than $5 \mathrm{~km} \mathrm{~s}^{-1}$, we applied an iterative $2 \sigma$ clipping procedure on the mean, until no stars could be eliminated as outliers. This was achieved in just four iterations, providing a catalogue of 26 stars, which are considered to be high confidence cluster members. Based on this sample of high confidence members, the average radial velocity of Pismis 18 becomes $-27.5 \mathrm{~km} \mathrm{~s}^{-1}$ with a standard deviation of $2.5 \mathrm{~km} \mathrm{~s}^{-1}$ and a median of $-27.8 \mathrm{~km} \mathrm{~s}^{-1}$. It is noted that no systematic differences were found between radial velocities derived with different setups, within the corresponding errors.

Among the 26 high confidence members, there are six stars observed with UVES, for which there are also detailed metal abundances, discussed in Sect. 7. Radial velocities for these six stars have also been obtained by Carlberg (2014) (see Col. 18 of Table 2). These values are in good agreement with our measurements within the quoted errors. A seventh UVES star in common with Carlberg (2014), CNAME13365001-6205376, also mentioned in Sect. 4.1, shows a discrepancy since it has $\mathrm{RV}=-27.3 \mathrm{~km} \mathrm{~s}^{-1}$ in Carlberg (2014) and $-18.4 \mathrm{~km} \mathrm{~s}^{-1}$ in our study. This discrepancy could be accounted for by the possible binary nature of this object. Although it might be a cluster member, it has not been included in the analysis of high confidence members.

In Fig. 3 we show the distribution of the radial velocities of the 20 GIRAFFE and six UVES high confidence member stars (blue and red histogram, respectively), as well as the radial veloc- 


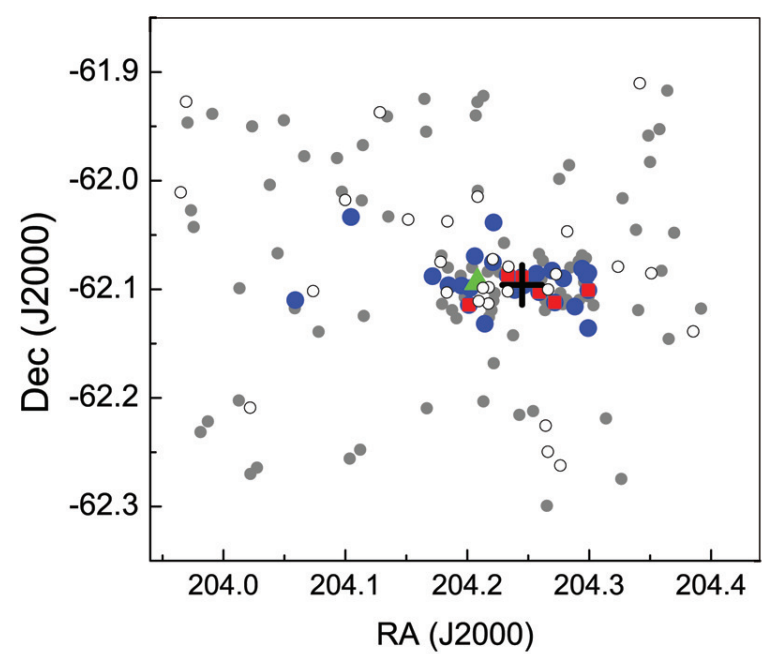

Fig. 4. Distribution of stars with assigned cluster membership, on the RA-Dec plane. With small grey filled circles we indicate all observed stars, with large blue filled circles the GIRAFFE radial velocity members, with red filled squares the UVES radial velocity members, with a green filled triangle the possible binary star discussed in the text and with a black cross we indicate the location of the cluster centre (as determined in Sect. 6). With open grey circles we indicate stars with $\mathrm{RV}$ s consistent with cluster membership, although their proper motions lie outside the 3- $\sigma$ acceptance radius discussed in Sect. 4.1 and shown in Fig. 2

ity distribution of all 142 observed stars (indicated in grey). The patterned bars correspond to the distribution of the radial velocities of Galactic stars in the direction of Pismis 18 according to the Besançon star count model for the Galaxy ${ }^{2}$ (Robin et al. 2003). The predicted distribution is scaled to the total number of observed stars divided by the number of stars in the VPHAS + photometry, in the same $V$ mag range and the same sky area. The scaled model predicts that $\simeq 60$ field stars fall within the selected boundaries of the cluster area, while 6-7 of these fall within the radial velocity range of the Pismis 18 members.

Figure 4 shows the location of the 26 radial velocity members on the RA-Dec plane. The stars assigned cluster membership according to their proper motion and radial velocity show a clear concentration close to the cluster centre, with the majority (24 of the 26) lying within a radius of $3.3 \mathrm{arcmin}$. The remaining two stars lie at distances up to $\simeq 5 \mathrm{arcmin}$ ( $3.6 \mathrm{pc}$ for the estimated distance of the cluster, see Sect. 6) from the cluster centre. Although the list of cluster members considered here is not exhaustive, as only high confidence members have been included, it seems that the radius of the cluster is close to 5 arcmin, similar to the estimate of Tadross (2008). Cantat-Gaudin et al. (2018a) have estimated that the radius enclosing half the high probability members (based on Gaia DR2 proper motions and parallaxes) is 2.88 arcmin. Following a similar approach, we derived a radius 1.48 arcmin enclosing half the high confidence members in our sample. This value is significantly lower than that of Cantat-Gaudin et al. (2018a) and remains unchanged if we consider all 35 proper motion members (i.e. without the RV selection). Apart from the small size of our sample, our targets were selected on the basis of their location on the CMD, without attempting a homogeneous spatial coverage of the field of view. Therefore, statistically the derivation of the radius is not very meaningful, and comparisons

2 http://model.obs-besancon.fr/ with values derived from larger and homogeneous samples are not informative.

It must be noted that proper motions are of paramount importance in assigning cluster membership, as there are several objects with radial velocities consistent with cluster membership (open grey circles in Fig. 4), but which are generally scattered over larger distances from the cluster centre and have inconsistent proper motion values. The average proper motion of the 26 high confidence members of Pismis 18 is pmra $=-5.65 \pm$

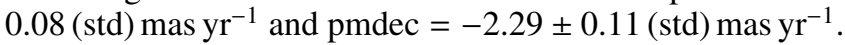

To summarize, six out of the ten UVES target stars and 20 out of the 132 GIRAFFE targets are considered as high probability cluster members on the basis of their proper motions, parallaxes and RV. The calculated cluster radial velocity dispersion of $2.5 \mathrm{~km} \mathrm{~s}^{-1}$ is higher than the $\simeq 1 \mathrm{~km} \mathrm{~s}^{-1}$ expected for a typical OC (e.g. Mermilliod et al. 2009). The larger dispersion could be caused by the fact that our sample consists mostly of upper main sequence stars which are more likely to rotate than the solar-type dwarf stars used by Mermilliod et al. (2009). If we use only the six UVES high confidence members lying on the red clump, the velocity dispersion falls to $0.7 \mathrm{~km} \mathrm{~s}^{-1}$.

\section{Atmospheric parameters}

The iDR 5 database provides effective temperatures, $T_{\text {eff }}$, for a total of 133 of the 142 observed stars, and surface gravities $(\log g)$ and metallicities $([\mathrm{Fe} / \mathrm{H}])$ for 123 of them. Metallicities based on GIRAFFE data are highly uncertain and therefore they are not used in our analysis. Of the 20 GIRAFFE high confidence radial members, 17 have measured $T_{\text {eff }}$ and 14 of those have $\log g$ measurements. We provide the atmospheric parameters for the high probability member stars in Table 3; we have kept the probable binary in this table and following ones even if its values were not used to compute cluster averages. Based on the six UVES members, the median metallicity of the cluster is $[\mathrm{Fe} / \mathrm{H}]=0.23 \pm 0.05$ dex. This value was adopted as the cluster metallicity in the cluster parameter determination described in the next section. It is noted that the metal abundance derived for the likely binary star is entirely consistent with the cluster value. It is noted that the global metallicity $[\mathrm{Fe} / \mathrm{H}]$ of Pismis 18 derived in the present work is higher than that given in previous papers that adopt the results of Gaia-ESO DR4 $(0.11 \pm 0.02$ and $0.10 \pm 0.03$, respectively, in Jacobson et al. 2016; Magrini et al. 2017). The difference is due to the improved process of homogenization and combination of Node results followed in iDR5. In particular, during iDR5 a lower number of Nodes participated in the analysis of the UVES WG11 spectra, compared to DR4. Most of the Nodes considered in the homogenization of $[\mathrm{Fe} / \mathrm{H}]$ for iDR5 based their derivations on the Equivalent Width (EW) analysis and tend to obtain a slightly higher $[\mathrm{Fe} / \mathrm{H}]$ than that obtained using spectral synthesis (see Jofré et al. (2017) for a discussion of systematic differences between different methods of metal abundance derivation). On the other hand, the $[\mathrm{Fe} / \mathrm{H}]$ and $[\mathrm{Fe} \mathrm{I} / \mathrm{H}]$ abundances recomputed by Nodes after the homogenization of the stellar parameters is much closer to the results of the DR 4 release (see Table 8, $0.04 \pm 0.06$ and $0.08 \pm 0.07$, respectively).

\section{Redetermined cluster parameters}

Using our membership assessment, we re-evaluated the fundamental parameters of Pismis 18. This evaluation has been based on a relatively small number of stars, spanning a relatively limited range in masses (upper main sequence and clump stars). 
However, as they are high confidence members, they provide a good comparison against other methods, which may be affected by contamination from field stars.

The centre of the cluster was determined to be at RA = $13^{\mathrm{h}} 36^{\mathrm{m}} 58.1^{\mathrm{s}}$, Dec $=-62^{\circ} 05^{\prime} 35^{\prime \prime}$ from the median values of the coordinates of the 26 high confidence members (Table 1: all coordinates are J2000). The right ascension agrees with all previous studies (including the WEBDA value) within $\sim 1$ ', except for the study of Piatti et al. (1998) where a large difference of about $7^{\prime}$ is noticed. In declination, the agreement with WEBDA and Tadross (2008) is excellent (within ten "), while there are discrepancies from two to seven arcmin with the other studies. The good agreement with the results of Tadross (2008) in both coordinates is encouraging in view of the different sample and method used.

The stellar population parameters (age, reddening, and de-reddened distance modulus) were derived by comparing Pisa theoretical isochrones (computed on purpose) with the recently made available Gaia DR2 photometry (Gaia Collaboration 2018b), by adopting the same maximum-likelihood technique described in detail in Randich et al. (2018). To show the robustness of the derived parameters we compared the cluster sequence obtained with the best fit set of parameters in several observational planes, using the $B V$-bands from Piatti et al. (1998), the $J H K_{s}$ (2MASS) and the gri (VPHAS+) photometry, along with the $G_{\mathrm{BP}}, G_{\mathrm{RP}}$ and $G$ Gaia magnitudes. All available photometric information is provided in Table 2 for all 142 observed stars (including the 26 high confidence members).

We computed stellar models using the Pisa evolutionary code (Degl'Innocenti et al. 2008; Tognelli et al. 2011; Dell'Omodarme et al. 2012), with the same input physics described in Randich et al. (2018) and Tognelli et al. (2018), except for the initial chemical composition, for which we adopted the value obtained from the six cluster members oberved with UVES (see Sect. 5). More specifically, we built a grid of models using three values of $[\mathrm{Fe} / \mathrm{H}]$, namely the fiducial value $[\mathrm{Fe} / \mathrm{H}]=+0.23 \mathrm{dex}$, and the two extremes (lower and upper limit) obtained assuming the estimated uncertainty $\Delta[\mathrm{Fe} / \mathrm{H}]= \pm 0.05 \mathrm{dex}$. We assumed the solar-scaled metal distribution given by Asplund et al. (2009; hereafter AS09), thus $[\alpha / \mathrm{Fe}]=0$, which is consistent with that measured for the six members observed with UVES (i.e. $[\alpha / \mathrm{Fe}]=+0.07 \pm 0.13$, see Sect. 7). In addition, we verified that for the adopted metallicity, a change in $[\alpha / \mathrm{Fe}]$ by \pm 0.1 has a negligible impact on the derived parameters. Adopting the AS09 solar-scaled metal distribution, a helium-to-metal enrichment ratio of two (Casagrande 2007) and a primordial helium abundance of $Y_{\mathrm{p}}=0.2485$ (Cyburt 2004), we derived an initial helium and metallicity of $(Y, Z)=(0.291,0.0212),(0.287,0.0191)$, and $(0.296,0.0235)$, for the fiducial, lower and upper $[\mathrm{Fe} / \mathrm{H}]$ values, respectively.

For the model computation we used our solar-calibrated mixing length parameter $\left(\alpha_{\mathrm{ML}}=2.0\right)$, which was assumed to be the same for stars with different masses and/or in different evolutionary stages. We included a step core overshooting in the models, for $M \geq 1.2 M_{\odot}$, with a standard value of $\beta_{\mathrm{ov}}=0.150$ (Valle et al. 2017). From the evolutionary tracks we obtained the isochrones in the age range $300 \mathrm{Myr}-2 \mathrm{Gyr}$ with a grid spacing of ten Myr, a good compromise between a dense enough and not extremely large grid to achieve a good age resolution.

To derive the cluster parameters, we opted for Gaia DR2 photometry, which has small uncertainties $(\Delta m(G)<0.001 \mathrm{mag}$ and $\Delta m(\mathrm{BP})$ and $\Delta m(\mathrm{RP})<0.01 \mathrm{mag})$, thus resulting in well constrained values for these parameters. To properly account for the extinction/reddening in the Gaia bands, we adopted the extinction law given in Eq. (1) in Gaia Collaboration (2018a). In addition to Gaia DR2 photometry, we also applied the same method using VPHAS + photometry. The derived parameters are fully compatible with those obtained using the Gaia DR2 photometry. We did not use the Piatti et al. (1998) BVI and the 2MASS photometry in the same manner, because, for the former the uncertainties were not available, while for the latter the CMD shows significant scatter leading to large uncertainties for the values of the derived parameters.

As already mentioned, we adopted the maximum-likelihood technique described in Randich et al. (2018), which they applied to young clusters in the TGAS catalogue. However, instead of assuming a fixed cluster distance based on TGAS parallaxes as was done in Randich et al. (2018), we treated the cluster distance as a free parameter, as Gaia DR2 parallaxes for relatively distant objects may suffer from non-negligible systematic errors of the order of 0.05 mas or more (see e.g. Cantat-Gaudin et al. 2018a; Riess et al. 2018; Stassun \& Torres 2018; Zinn et al. 2018). Such a systematic error would affect significantly the cluster distance and, as a result, the quality of the isochrone fitting, as is further discussed later.

We recall that in the adopted maximum-likelihood technique the best values of the vector of cluster parameters (age, reddening, distance $)=\left(\tau, E(B-V), \mathrm{DM}_{0}\right)$ are estimated together. To properly evaluate the confidence interval (hereafter CI) of such quantities, we adopted a Monte Carlo procedure. We perturbed independently the photometric data for each star in each band using the available information on the uncertainty (which we assumed to be Gaussian) to obtain $N$ representations of the same cluster. We set $N=100$, which is large enough to guarantee convergence. Thus, for each perturbed sample $j$ we derived the vector $\left.\left(\tau_{j}, E(B-V)_{j}, \mathrm{DM}_{0, j}\right)\right)$. The best value for each one of the parameters and its CI were obtained from the ordered sample of the $N$ simulations, by taking the mid value of the distribution (best value), and the 16 and 84 percentile (which define the confidence interval, i.e. the uncertainty). This approach could account only for the observational uncertainties on the photometric bands. However, we wanted to give an estimation of the uncertainties in $\tau, E(B-V)$ and $\mathrm{DM}_{0}$ due to the adopted chemical composition. To this purpose, we computed models for the upper and lower limit of $[\mathrm{Fe} / \mathrm{H}]$ and used these two grids to re-derive the cluster parameters and their CI (using Monte Carlo simulations for the photometry). The effect of adopting a different chemical composition was to shift the best values of the derived parameters with respect to that estimated using the fiducial value of $[\mathrm{Fe} / \mathrm{H}]$. We assumed this shift to be representative of the errors due to $\Delta[\mathrm{Fe} / \mathrm{H}]$, and incorporated in the errors caused by photometry alone. We found that $\Delta[\mathrm{Fe} / \mathrm{H}]$ accounted for about one half of the uncertainty on the estimated age, reddening and distance modulus.

We show the comparisons between the best set of isochrones (best $\tau, E\left(B-V\right.$ and $\left.\mathrm{DM}_{0}\right)$ and data in several photometric bands in the panels of Fig. 5. Our best estimate led to an age of $\tau=700_{-50}^{+40} \mathrm{Myr}$, a reddening of $E(B-V)=0.562_{-0.026}^{+0.012} \mathrm{mag}$ and a de-reddened distance modulus of $\mathrm{DM}_{0}=11.96_{-0.24}^{+0.10} \mathrm{mag}$ (i.e. a mean distance of $2.47_{-0.26}^{+0.11} \mathrm{kpc}$ and a mean parallax of $0.406_{-0.019}^{+0.047}$ mas). The quoted distance error encompasses the uncertainties related to our choice of stellar models. Indeed, a quick independent interactive isochrone fit with different sets of evolutionary models (PARSEC, BaSTI and Dartmouth) confirmed the given distance modulus within $0.1 \mathrm{mag}$. As a general comment, we note that the best isochrone achieved a very good agreement with the data in all the adopted photomet- 
Table 3. Atmospheric parameters for high confidence members.

\begin{tabular}{llrrrrrrrr}
\hline \hline CNAME & $T_{\text {eff }}$ & Err & $\log g$ & Err & {$[\mathrm{Fe} / \mathrm{H}]$} & Err & $\xi$ & Err & Setup $^{(1)}$ \\
\hline $13364831-6206517$ & 4983 & 60 & 2.85 & 0.12 & 0.23 & 0.10 & 1.545 & 0.046 & $U$ \\
$13365597-6205130$ & 4882 & 60 & 2.62 & 0.12 & 0.23 & 0.10 & 1.655 & 0.127 & $U$ \\
$13365882-6205197$ & 5045 & 60 & 3.01 & 0.12 & 0.14 & 0.10 & 1.695 & 0.174 & $U$ \\
$13370214-6206095$ & 4933 & 60 & 2.81 & 0.12 & 0.29 & 0.10 & 1.565 & 0.104 & $U$ \\
$13370523-6206433$ & 4861 & 60 & 2.64 & 0.12 & 0.22 & 0.10 & 1.655 & 0.156 & $U$ \\
$13371182-6206030$ & 4950 & 60 & 2.74 & 0.11 & 0.21 & 0.10 & 1.760 & 0.082 & $U$ \\
$13365001-6205376$ & 4955 & 60 & 2.81 & 0.11 & 0.22 & 0.10 & 1.390 & 0.026 & $U$ \\
$13361412-6206360$ & 7750 & 51 & & & & & & & $G$ \\
$13362510-6202004$ & & & & & & & & & $G$ \\
$13364117-6205166$ & 8520 & 750 & 3.55 & 0.45 & & & & & $G$ \\
$13364430-6205471$ & 7022 & 120 & 4,37 & 0.16 & & & & & $G$ \\
$13364687-6205483$ & 8981 & 750 & 3.96 & 0.30 & & & & & $G$ \\
$13364855-6205555$ & 7498 & 96 & 4.25 & 0.21 & & & & & $G$ \\
$13364946-6204100$ & 6633 & 148 & 4.21 & 0.14 & & & & & $G$ \\
$13365149-6207542$ & & & & & & & & & $G$ \\
$13365304-6204298$ & 6791 & 102 & 4.23 & 0.13 & & & & & $G$ \\
$13365318-6202181$ & 8378 & 750 & 3.7 & 0.45 & & & & & $G$ \\
$13365737-6206023$ & 8613 & 750 & 3.72 & 0.45 & & & & & $G$ \\
$13365917-6205472$ & & & & & & & & & $G$ \\
$13370162-6205086$ & 7961 & 62 & & & & & & & $G$ \\
$13370473-6204579$ & 8077 & 750 & 3.5 & 0.45 & & & & & $G$ \\
$13370693-6205236$ & 8801 & 750 & 3.80 & 0.30 & & & & & $G$ \\
$13370918-6206569$ & 8779 & 750 & 4.12 & 0.20 & & & & & \\
$13371063-6204512$ & 7004 & 500 & 3.72 & 0.45 & & & & & $G$ \\
$13371147-6205141$ & 7121 & 92 & & & & & & & $G$ \\
$13371180-6208085$ & 8613 & 750 & 3.63 & 0.45 & & & & & $G$ \\
$13371184-6205052$ & 7968 & 750 & 3.00 & 0.70 & & & & & $G$ \\
\hline
\end{tabular}

Notes. ${ }^{(1)} \mathrm{U}: U V E S$, G: GIRAFFE; ${ }^{(2)}$ possible binary star.

ric bands for both $\mathrm{MS}$ and $\mathrm{RC}$ stars, with the exception of the 2MASS CMD which shows large scatter; however, even in this case, the best isochrone could reproduce the RC stars. As discussed in Sect. 5, the metallicity of Pismis 18 according to GES DR4 is significantly lower (around $[\mathrm{Fe} / \mathrm{H}]=$ +0.10 dex) than the value derived in the present paper. Adopting this lower metallicity and applying the same procedure, we derived a distance modulus of $\mathrm{DM}_{0}=11.91 \mathrm{mag}$, which is $0.05 \mathrm{mag}$ lower than the one obtained for $[\mathrm{Fe} / \mathrm{H}]=+0.23$, but within the formal uncertainties. Similarly, the derived age was reduced by ten Myr, again within the quoted errors. However, the reddening value obtained was higher by about 0.038 mag, which is about $3 \sigma$ larger than the value for obtained for $[\mathrm{Fe} / \mathrm{H}]=0.23$.

The redetermined parameter values given in the last column of Table 1 are in good agreement with previous studies. The reddening we found is compatible within the uncertainties with the values given by Piatti et al. (1998) and Kharchenko et al. (2013) and a bit lower than that derived by Tadross (2008), who however does not give the associated uncertainty. Also the distance is in very good agreement with that given in Piatti et al. (1998) and Kharchenko et al. (2013), while that found by Tadross (2008) is much smaller than the others. The ages given in the literature are a bit larger than that we found in the present work. We note that the larger age (still consistent within the errors) adopted by Piatti et al. (1998) is due to their averaging the results obtained from isochrone fitting and integrated spectroscopy. Their photometry-based age is about $0.9 \mathrm{Gyr}$, in reasonable agreement with our findings.
We also show a comparison between our best fit isochrone and the data in the $\left(\log T_{\text {eff }}, \log g\right)$ plane (see Fig. 6). The relatively large scatter displayed by the MS stars is expected as in this case the atmospheric parameters (given in Table 3) were derived from GIRAFFE low resolution spectra. Despite this scatter, the best isochrone is fully compatible with the data for both MS and RC stars (for which the atmospheric parameters are much better constrained, as they are based on high resolution UVES spectra).

The location of the RC stars on the CMDs strongly affects the distance determination and limits the acceptable values of $\mathrm{DM}_{0}$. The uncertainty in $\mathrm{DM}_{0}$ is asymmetric around the best value. The best value of the distance modulus is obtained when using the upper part of the central helium burning phase. Adopting a larger distance would cause these RC stars to move away from the isochrone, thus producing a worse fit. Another possible solution would be to fit such stars with the lower part of the theoretical RC, which is still acceptable in the fitting procedure and gives a relatively good quality fit. This latter case corresponds to a smaller distance. Thus, this simple statement should qualitatively explain the asymmetry in the uncertainty on $\mathrm{DM}_{0}$. Another point concerns the large discrepancy between the parallax provided by Gaia DR2 and what we derived here (similar to that used in the literature). We found a mean parallax of $0.406_{-0.019}^{+0.047}$ mas (corresponding to $2.47 \mathrm{pc}$ ), to be compared with the $0.338 \pm 0.043$ mas (corresponding to $2.94 \mathrm{kpc}$ ) we derived using the Gaia parallaxes. The difference between the two determinations is about 0.068 mas. We tried to use the Gaia distance as a prior in our isochrone fitting, but the 
Table 4. UVES members light and $\alpha$-element abundances.

\begin{tabular}{|c|c|c|c|c|c|c|c|c|c|c|}
\hline CNAME & Li I & $\mathrm{NaI}$ & $\mathrm{Mg}_{\mathrm{I}}$ & $\mathrm{A} l_{\mathrm{I}}$ & Si I & Si II & Ca I & $\mathrm{Ca}$ II & Ti I & Ti II \\
\hline $13364831-6206517$ & & & $7.58 \pm 0.14$ & $6.46 \pm 0.06$ & $7.66 \pm 0.08$ & $7.57 \pm 0.13$ & $6.48 \pm 0.08$ & & $4.92 \pm 0.08$ & $5.08 \pm 0.11$ \\
\hline & & & & & & & & & & \\
\hline & 1 & .06 & .17 & & & 0.16 & $6.42 \pm$ & & & \\
\hline $4-6206095$ & 0 & .05 & & & & 0.18 & $6.47 \pm$ & $6.37 \pm 0.09$ & & \\
\hline & & & & & & & & & & \\
\hline $13371182-6206030$ & $58 \pm 0.07$ & .10 & $7.60 \pm$ & & $7.69 \pm 0$ & $7.51 \pm$ & 6.46 & $6.12 \pm 0.09$ & & $5.08 \pm 0.14$ \\
\hline $13365001-6205376$ & $1.34 \pm 0.05$ & $6.48 \pm 0.07$ & $7.58 \pm 0.09$ & $6.44 \pm 0.05$ & $7.67 \pm 0.08$ & $7.64 \pm 0.08$ & $6.49 \pm 0.06$ & $6.26 \pm 0.01$ & $4.93 \pm 0.09$ & $5.09 \pm 0.11$ \\
\hline
\end{tabular}

Table 5. Iron-peak abundances for UVES members.

\begin{tabular}{|c|c|c|c|c|c|c|c|c|c|c|c|c|}
\hline CNAME & $\mathrm{Sc}$ I & $\mathrm{Sc}$ II & $\mathrm{V}_{\mathrm{I}}$ & $\mathrm{Cr} I$ & CriI & Mn I & $\mathrm{Fe}_{\mathrm{I}}$ & $\mathrm{Fe}_{\text {II }}$ & CoI & $\mathrm{Ni}$ I & $\mathrm{Cu}$ I & $\mathrm{Zn} I$ \\
\hline $13364831-6206517$ & $3.26 \pm 0.07$ & $3.43 \pm 0.09$ & $4.11 \pm 0.07$ & $5.69 \pm 0.10$ & $5.71 \pm 0.10$ & $5.50 \pm 0.05$ & $7.53 \pm 0.07$ & $7.63 \pm 0.09$ & $4.98 \pm 0.08$ & $6.31 \pm 0.10$ & $4.11 \pm 0.06$ & $4.66 \pm 0.03$ \\
\hline $13365597-6205130$ & $3.19 \pm 0.06$ & $3.39 \pm 0.12$ & $4.06 \pm 0.09$ & $5.64 \pm 0.11$ & $5.69 \pm 0.11$ & $5.48 \pm 0.05$ & $7.50 \pm 0.07$ & $7.59 \pm 0.07$ & $4.96 \pm 0.05$ & $6.30 \pm 0.11$ & $4.16 \pm 0.09$ & $4.51 \pm 0.01$ \\
\hline $13365882-6205197$ & $3.28 \pm 0.07$ & $3.37 \pm 0.23$ & $4.13 \pm 0.09$ & $5.68 \pm 0.12$ & $5.69 \pm 0.16$ & $5.46 \pm 0.08$ & $7.45 \pm 0.09$ & $7.55 \pm 0.11$ & $4.94 \pm 0.12$ & $6.23 \pm 0.13$ & $4.13 \pm 0.10$ & $4.53 \pm 0.19$ \\
\hline $13370214-6206095$ & $3.22 \pm 0.07$ & $3.44 \pm 0.19$ & $4.09 \pm 0.08$ & $5.69 \pm 0.10$ & $5.77 \pm 0.10$ & $5.48 \pm 0.09$ & $7.52 \pm 0.08$ & $7.66 \pm 0.08$ & $4.99 \pm 0.07$ & $6.32 \pm 0.09$ & $4.25 \pm 0.10$ & $4.55 \pm 0.01$ \\
\hline $13370523-6206433$ & $3.16 \pm 0.09$ & $3.32 \pm 0.09$ & $4.01 \pm 0.09$ & $5.61 \pm 0.10$ & $5.73 \pm 0.05$ & $5.41 \pm 0.09$ & $7.44 \pm 0.08$ & $7.58 \pm 0.11$ & $4.91 \pm 0.06$ & $6.23 \pm 0.12$ & $4.20 \pm 0.14$ & $4.41 \pm 0.11$ \\
\hline $13371182-6206030$ & $3.31 \pm 0.06$ & $3.42 \pm 0.14$ & $4.18 \pm 0.08$ & $5.69 \pm 0.11$ & $5.72 \pm 0.06$ & $5.53 \pm 0.06$ & $7.53 \pm 0.08$ & $7.58 \pm 0.09$ & $5.03 \pm 0.06$ & $6.33 \pm 0.10$ & $4.25 \pm 0.16$ & $4.44 \pm 0.10$ \\
\hline $13365001-6205376$ & $3.26 \pm 0.06$ & $3.42 \pm 0.13$ & $4.12 \pm 0.08$ & $5.67 \pm 0.11$ & $5.71 \pm 0.09$ & $5.47 \pm 0.07$ & $7.53 \pm 0.07$ & $7.63 \pm 0.09$ & $4.97 \pm 0.05$ & $6.33 \pm 0.10$ & $4.19 \pm 0.09$ & $4.72 \pm 0.08$ \\
\hline
\end{tabular}

Table 6. UVES members neutron-capture element abundances.

\begin{tabular}{lcccccccc}
\hline \hline CNAME & Y $_{\text {II }}$ & $\mathrm{Zr}_{\text {I }}$ & $\mathrm{Zr}_{\text {II }}$ & $\mathrm{Ba}_{\text {II }}$ & La II & $\mathrm{Ce}_{\text {II }}$ & $\mathrm{Nd}_{\text {II }}$ & Eu II \\
\hline $13364831-6206517$ & $2.32 \pm 0.07$ & $2.63 \pm 0.04$ & $2.84 \pm 0.10$ & $2.41 \pm 0.04$ & $1.12 \pm 0.08$ & $1.97 \pm 0.05$ & $1.71 \pm 0.08$ & $0.53 \pm 0.02$ \\
$13365001-6205376$ & $2.32 \pm 0.08$ & $2.63 \pm 0.05$ & $2.75 \pm 0.07$ & $2.40 \pm 0.04$ & $1.01 \pm 0.03$ & $1.86 \pm 0.01$ & $1.64 \pm 0.13$ & $0.55 \pm 0.02$ \\
$13365597-6205130$ & $2.25 \pm 0.07$ & $2.55 \pm 0.03$ & $2.69 \pm 0.05$ & $2.26 \pm 0.08$ & $1.09 \pm 0.07$ & $1.76 \pm 0.02$ & $1.65 \pm 0.08$ & $0.58 \pm 0.02$ \\
$13365882-6205197$ & $2.20 \pm 0.14$ & $2.66 \pm 0.05$ & $2.75 \pm 0.14$ & $2.27 \pm 0.08$ & $1.06 \pm 0.07$ & $1.65 \pm 0.05$ & $1.65 \pm 0.08$ & $0.53 \pm 0.02$ \\
$13370214-6206095$ & $2.37 \pm 0.09$ & $2.57 \pm 0.06$ & $2.80 \pm 0.08$ & $2.43 \pm 0.02$ & $1.09 \pm 0.05$ & $1.91 \pm 0.01$ & $1.70 \pm 0.10$ & $0.57 \pm 0.03$ \\
$13370523-6206433$ & $2.22 \pm 0.08$ & $2.50 \pm 0.06$ & $2.78 \pm 0.09$ & $2.31 \pm 0.03$ & $1.03 \pm 0.09$ & $1.72 \pm 0.02$ & $1.62 \pm 0.09$ & $0.51 \pm 0.02$ \\
$13371182-6206030$ & $2.31 \pm 0.07$ & $2.68 \pm 0.06$ & $2.79 \pm 0.08$ & $2.31 \pm 0.08$ & $1.17 \pm 0.08$ & $1.85 \pm 0.02$ & $1.73 \pm 0.07$ & $0.58 \pm 0.01$ \\
\hline
\end{tabular}

results were not satisfactory. The isochrones tended to drastically underestimate the magnitude of the MS stars, while the TurnOff region and the RC were not well reproduced. Therefore, it seems very unlikely that the cluster could have such a large distance. It is noted that similar discrepancies have been reported for distant clusters (Lindegren et al. 2018). Such systematic effects are expected to be minimized at the end of the Gaia mission.

\section{The chemical composition of Pismis $\mathbf{1 8}$}

Tables $4-6$ give elemental abundances in the form $12+\log (\mathrm{X} / \mathrm{H})$ of six high confidence radial velocity members (plus the suspect binary) observed with UVES for light, $\alpha$, Fe-peak, and neutroncapture elements. For each star, its elemental abundances were computed by combining the results of different nodes for each absorption line. The results of all lines of the same element are then combined to produce the final abundance per element per star. The corresponding errors were computed from lineby-line abundance variations, after combining the abundances of the various nodes (see Smiljanic et al. 2014 for a detailed description).

In Table 7 we show the Solar reference abundances by Grevesse et al. (2007), the GES iDR5 Solar abundances and the median abundances of M 67 giant stars. To compute the $[\mathrm{X} / \mathrm{H}]$ ratios we adopted the Solar abundance scale of GES iDR5, using for most elements the homogenized Solar abundances provided in the final iDR5 table. For elements that are not measured in the Solar spectra (Nd and $\mathrm{Eu}$ ) and for $\mathrm{La}$, we adopted the abun-
Table 7. Reference element abundances.

\begin{tabular}{lcccc}
\hline \hline Element & $\begin{array}{c}\text { Atomic } \\
\text { number }\end{array}$ & $\begin{array}{c}\text { Grevesse et al. } \\
(2007)\end{array}$ & idR5-solar & M67 \\
\hline $\mathrm{Na}$ & 11 & $6.17 \pm 0.04$ & $6.17 \pm 0.05$ & \\
$\mathrm{Mg}$ & 12 & $7.53 \pm 0.09$ & $7.51 \pm 0.07$ & \\
$\mathrm{Al}$ & 13 & $6.37 \pm 0.06$ & $6.34 \pm 0.04$ & \\
$\mathrm{Si}$ & 14 & $7.51 \pm 0.04$ & $7.48 \pm 0.06$ & \\
$\mathrm{Ca}$ & 20 & $6.31 \pm 0.04$ & $6.31 \pm 0.12$ & \\
$\mathrm{Sc}$ & 21 & $3.17 \pm 0.10$ & $3.27 \pm 0.06$ & \\
$\mathrm{Ti}$ & 22 & $4.90 \pm 0.06$ & $4.90 \pm 0.08$ & \\
$\mathrm{~V}$ & 23 & $4.00 \pm 0.02$ & $4.00 \pm 0.09$ & \\
$\mathrm{Cr}$ & 24 & $5.64 \pm 0.10$ & $5.61 \pm 0.09$ & \\
$\mathrm{Mn}$ & 25 & $5.39 \pm 0.03$ & $5.39 \pm 0.06$ & \\
$\mathrm{Fe}$ & 26 & $7.45 \pm 0.05$ & $7.47 \pm 0.06$ & \\
$\mathrm{Co}$ & 27 & $4.92 \pm 0.08$ & $4.83 \pm 0.08$ & \\
$\mathrm{Ni}$ & 28 & $6.23 \pm 0.04$ & $6.23 \pm 0.07$ & \\
$\mathrm{Cu}$ & 29 & $4.21 \pm 0.04$ & $4.12 \pm 0.10$ & \\
$\mathrm{Zn}$ & 30 & $4.60 \pm 0.03$ & $4.60 \pm 0.06$ & \\
$\mathrm{Y}$ & 39 & $2.21 \pm 0.02$ & $2.19 \pm 0.12$ & \\
$\mathrm{Zr}$ & 40 & $2.58 \pm 0.02$ & $2.53 \pm 0.13$ & \\
$\mathrm{Ba}$ & 56 & $2.17 \pm 0.07$ & $2.17 \pm 0.06$ & \\
$\mathrm{La}$ & 57 & $1.13 \pm 0.05$ & & $0.97 \pm 0.07$ \\
$\mathrm{Ce}$ & 58 & $1.70 \pm 0.10$ & $1.70 \pm 0.11$ & \\
$\mathrm{Nd}$ & 60 & $1.45 \pm 0.05$ & & $1.56 \pm 0.02$ \\
$\mathrm{Eu}$ & 63 & $0.52 \pm 0.06$ & & $0.42 \pm 0.01$ \\
\hline
\end{tabular}

dances of member giant stars in the calibration open cluster M 67, which is known to have a chemical composition very close 

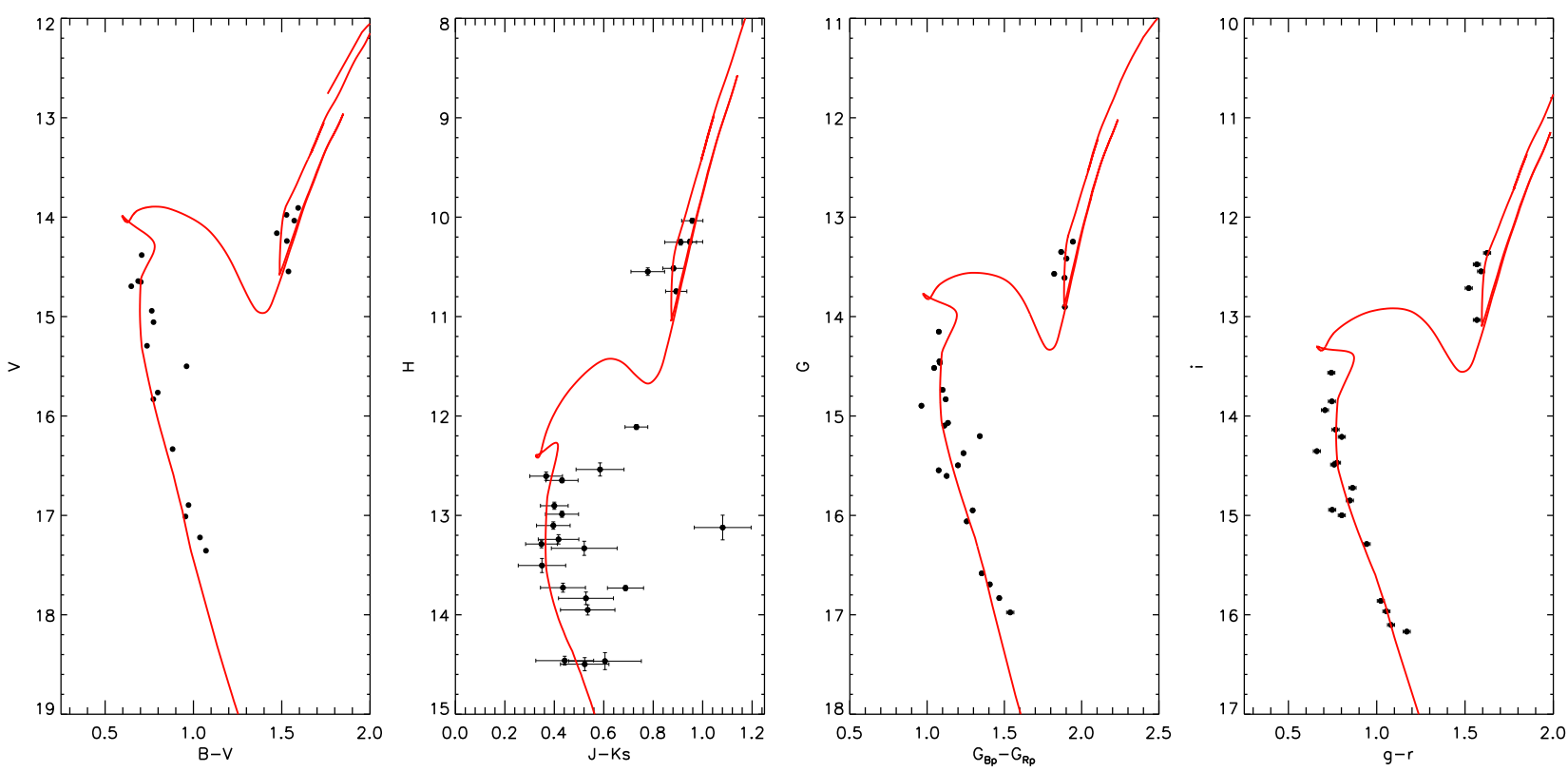

Fig. 5. Comparison between the Pisa best isochrone (corresponding to $\tau=700_{-50}^{+40} \mathrm{Myr}, E(B-V)=0.562_{-0.026}^{+0.012} \mathrm{mag}$ and $\mathrm{DM}_{0}=11.96_{-024}^{+0.10} \mathrm{mag}$ ) and the observations in several photometric bands, namely $B V$ (Piatti et al. 1998), $J H K_{s}$ (2MASS), $G_{\mathrm{BP}} G_{\mathrm{RP}} G$ (Gaia DR2), and gri (VPHAS+). The uncertainties in magnitude and colour are too small to show on the two right-most diagrams. No uncertainties were available for the BV photometry.

Table 8. Cluster average element abundances.

\begin{tabular}{|c|c|c|c|}
\hline Element & Abundance & {$[\mathrm{X} / \mathrm{H}]$} & {$[\mathrm{X} / \mathrm{Fe}]$} \\
\hline $\mathrm{Na}$ & $6.50 \pm 0.02$ & $0.33 \pm 0.05$ & $0.31 \pm 0.11$ \\
\hline $\operatorname{Mg}_{I}$ & $7.59 \pm 0.02$ & $0.08 \pm 0.07$ & $0.04 \pm 0.16$ \\
\hline $\mathrm{Al}$ I & $6.44 \pm 0.01$ & $0.10 \pm 0.04$ & $0.08 \pm 0.13$ \\
\hline $\mathrm{Si}$ I & $7.68 \pm 0.01$ & $0.20 \pm 0.06$ & $0.16 \pm 0.13$ \\
\hline $\mathrm{CaI}$ & $6.45 \pm 0.01$ & $0.14 \pm 0.12$ & $0.11 \pm 0.13$ \\
\hline Sc II & $3.41 \pm 0.05$ & $0.14 \pm 0.08$ & $0.10 \pm 0.16$ \\
\hline Ti I & $4.91 \pm 0.01$ & $0.00 \pm 0.08$ & $-0.05 \pm 0.13$ \\
\hline $\mathrm{V}_{\mathrm{I}}$ & $4.10 \pm 0.01$ & $0.10 \pm 0.09$ & $0.04 \pm 0.13$ \\
\hline CrI & $5.69 \pm 0.01$ & $0.08 \pm 0.09$ & $0.03 \pm 0.15$ \\
\hline $\mathrm{Mn}$ I & $5.48 \pm 0.02$ & $0.09 \pm 0.06$ & $0.06 \pm 0.12$ \\
\hline $\mathrm{Fe}_{\mathrm{I}}$ & $7.51 \pm 0.01$ & $0.04 \pm 0.06$ & \\
\hline $\mathrm{Fe}$ II & $7.59 \pm 0.01$ & $0.08 \pm 0.07$ & \\
\hline Co I & $4.97 \pm 0.02$ & $0.14 \pm 0.08$ & $0.11 \pm 0.12$ \\
\hline Ni I & $6.31 \pm 0.01$ & $0.07 \pm 0.07$ & $0.03 \pm 0.15$ \\
\hline $\mathrm{Cu}$ I & $4.18 \pm 0.03$ & $0.06 \pm 0.11$ & $0.05 \pm 0.14$ \\
\hline $\mathrm{Zn}$ I & $4.52 \pm 0.07$ & $0.06 \pm 0.07$ & $-0.11 \pm 0.12$ \\
\hline $\mathrm{Y}_{\mathrm{II}}$ & $2.38 \pm 0.02$ & $0.09 \pm 0.12$ & $0.06 \pm 0.13$ \\
\hline Zr II & $2.79 \pm 0.03$ & $0.26 \pm 0.13$ & $0.23 \pm 0.13$ \\
\hline Ba II & $2.31 \pm 0.03$ & $0.14 \pm 0.07$ & $0.15 \pm 0.12$ \\
\hline La II & $1.09 \pm 0.01$ & $0.12 \pm 0.06$ & $0.09 \pm 0.13$ \\
\hline $\mathrm{Ce}_{\text {II }}$ & $1.80 \pm 0.02$ & $0.10 \pm 0.11$ & $0.07 \pm 0.10$ \\
\hline $\mathrm{Nd}$ II & $1.68 \pm 0.01$ & $0.12 \pm 0.01$ & $0.09 \pm 0.13$ \\
\hline Eu II & $0.55 \pm 0.01$ & $0.13 \pm 0.01$ & $0.11 \pm 0.10$ \\
\hline
\end{tabular}

to Solar (see, e.g. Tautvaišiene et al. 2000; Shetrone \& Sandquist 2000; Yong et al. 2005; Randich et al. 2006; Pace et al. 2008; Önehag et al. 2014; Bertelli Motta et al. 2017).

The median abundances of Pismis 18 (for 22 elements, i.e. other than $\mathrm{Li}$ ) and their standard deviations based on the six member stars are provided in Col. 2 of Table 8. In Cols. 3 and 4 , we present the median $[\mathrm{X} / \mathrm{H}]$ and $[\mathrm{X} / \mathrm{Fe}]$ abundance ratios of Pismis 18 (the latter were calculated using $\mathrm{Fe}$ I and not $[\mathrm{Fe} / \mathrm{H}]$, see discussion in Sect. 5).

\subsection{Light elements}

\subsubsection{Lithium}

Lithium was measured in stars along the MS and in all RC stars. Figure 7 gives the dependence of $\mathrm{Li}$ abundance on $T_{\text {eff }}$, that is on evolutionary phase. All Li-rich stars, and all upper limits, are MS stars. Instead, all of the six evolved members have true measurements, not upper limits. Their values are all low, as expected from their evolutionary phase, after the first dredge-up. The star just below the RC and the probable binary have the highest $\mathrm{Li}$ abundances (Table 4) even if they do not qualify as "Li-rich" in the absolute sense (see e.g. the Li-rich stars discussed by Smiljanic et al. 2018a, discovered among GES targets)

\subsubsection{Sodium}

Sodium is the only elemental abundance which is significantly super-solar, with $[\mathrm{Na} / \mathrm{Fe}]=+0.31$. However, this is the value in LTE, and NLTE corrections for RC stars are about -0.1 dex (see, e.g. Smiljanic et al. 2016). A similar overabundance in giant stars (it is noted that the UVES targets are evolved stars) has been routinely observed and can be attributed to evolutionary mixing of products of the Ne-Na cycle during the first dredge-up phase. Smiljanic et al. (2016) discuss this using GES clusters, showing that a value of about 0.2 dex is normal for clusters of similar age/turnoff mass (see their Table 2 and Fig. 5).

\section{2. $\alpha$-elements}

As far as $\alpha$-elements $(\mathrm{Mg}, \mathrm{Si}, \mathrm{Ca}, \mathrm{Ti}$ - even if only the first traces exclusively massive-stars nucleosynthesis, while others, especially $\mathrm{Ti}$, come from several channels) are concerned, the abundances over $\mathrm{H}$ measured for Pismis 18 are close to solar values or slightly over-abundant (see Table 8, for instance $[\mathrm{Si} \mathrm{I} / \mathrm{H}]=0.20 \pm 0.06)$. Also $[\mathrm{Mg} / \mathrm{Fe}],[\mathrm{Ca} / \mathrm{Fe}],[\mathrm{SI} / \mathrm{Fe}]$ and $[\mathrm{Ti} / \mathrm{Fe}]$ range from slightly sub-solar to super-solar, with a mean value of $[\alpha / \mathrm{Fe}]=0.07 \pm 0.13$. The individual abundances and abundance ratios are provided in Table 8. 


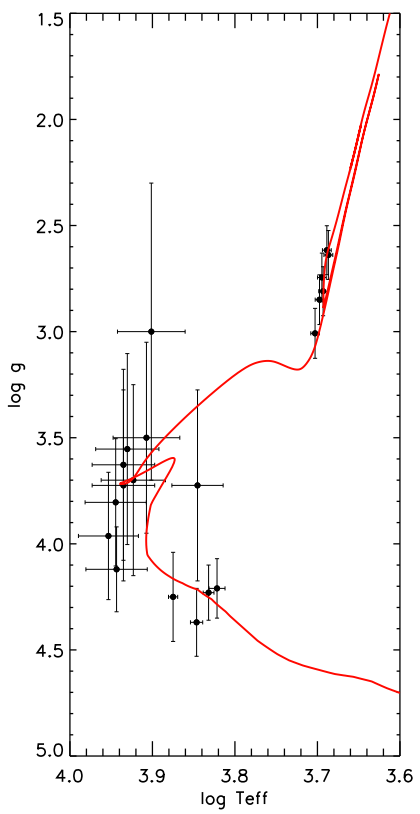

Fig. 6. Comparison between the Pisa best fit isochrone (corresponding to $\tau=700_{-50}^{+40} \mathrm{Myr}, E(B-V)=0.562_{-0.026}^{+0.012} \mathrm{mag}$ and $\mathrm{DM}_{0}=$ $\left.11.96_{-0.24}^{+0.10} \mathrm{mag}\right)$ and the determined $\log T_{\text {eff }}$ and $\log g$ values.

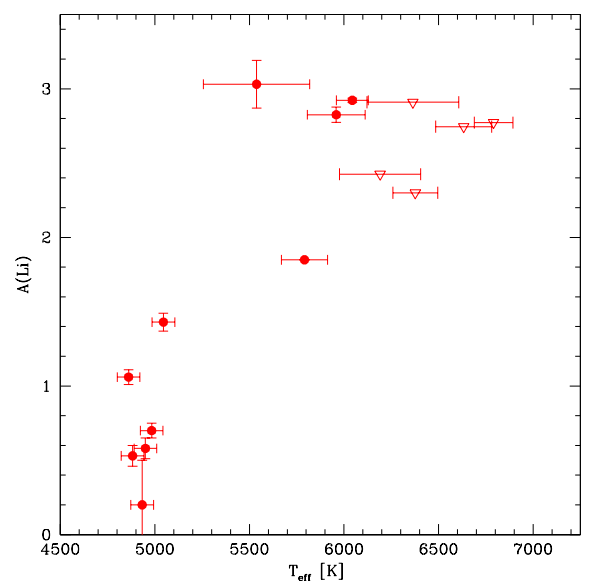

Fig. 7. Abundance of $\mathrm{Li}$ as a function of temperature (filled symbols with error bars indicate specific measured values, while empty triangles indicate upper limits.

Due to the different timescales involved in the production of the $\alpha$ elements and of iron and to the different star formation rates in the inner and outer part of the Galactic discthe so-called inside-out formation of the disc- classical chemical evolution models predict a depletion of the $[\alpha / \mathrm{Fe}]$ ratio in the inner disc coupled with an enhancement in the outskirts. This is indeed predicted by several chemical evolution models (see, e.g. Magrini et al. 2009, 2015; Kubryk et al. 2013; Minchev et al. 2014). For this reason, it would be expected that clusters located in the inner disc should present a depletion in $\alpha$-elements over iron with respect to solar values. However, observations of young populations, as shown for instance by Chiappini et al. (2015), Martig et al. (2015), Magrini et al. (2017), and Casamiquela et al. (2018) seem to contradict the expectations of chemical evolution models built on an insideout scenario (see for example Fig. 10 of Minchev et al. 2014). However, the point is that these results do not invalidate the inside-out formation of the disc, but indicate that the nucleosynthesis of some elements is more complex than believed

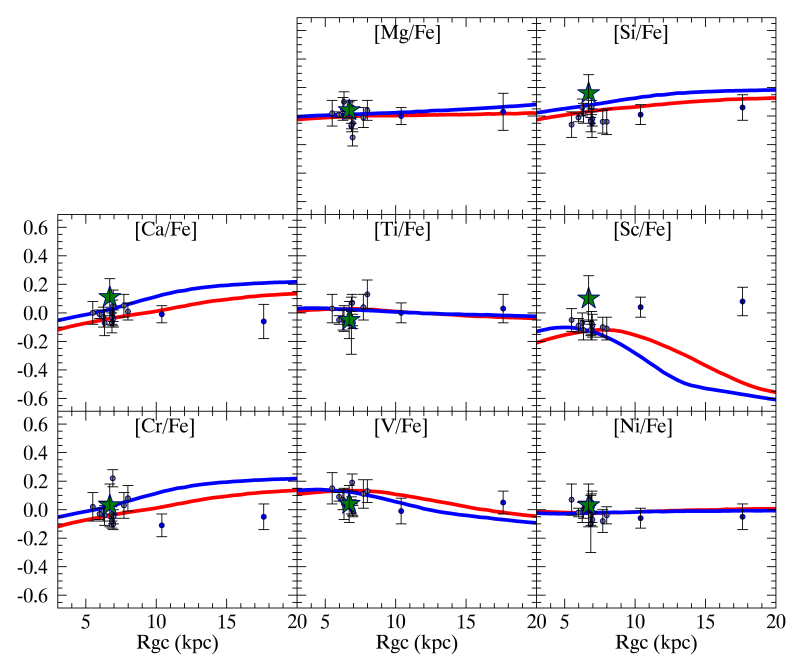

Fig. 8. Abundance ratios as a function of galactocentric distance $\left(R_{\mathrm{GC}}\right)$ for Pismis 18 (green star) compared with the results of Magrini et al. (2017) for both clusters (grey circles - the youngest clusters, age $<2 \mathrm{Gyr}$, and in blue the oldest ones) and models (red-present time- and blue - 5 Gyr ago - curves).

in the past. In particular, Chiappini (2005) already pointed out the $\mathrm{Mg}$ problem concluding that larger quantities of $\mathrm{Mg}$ should have been produced at recent epochs to explain the trend of $[\mathrm{Mg} / \mathrm{Fe}]$ vs. $[\mathrm{Fe} / \mathrm{H}]$. This can be achieved either by different SNIa models or by SNII metallicity dependent yields, as done in Magrini et al. (2017). Pismis 18, which is located at $\simeq 6.8 \mathrm{kpc}$ from the Galactic Centre, allows us to check the presence or not of such depletion. With $[\alpha / \mathrm{Fe}]=0.07 \pm 0.13$ it is in agreement, within the errors, with both the model predictions and the observations of the GES DR 4 clusters shown in Fig. 8 of Magrini et al. (2017).

\subsection{Iron-peak elements}

We measured the abundances of several iron-peak elements such as $\mathrm{Sc}, \mathrm{V}, \mathrm{Cr}, \mathrm{Mn}, \mathrm{Co}$, and $\mathrm{Ni}$, whose abundance ratios are all roughly solar. For some of them we could compare the theoretical and observational results of Magrini et al. (2017). In Fig. 8, we compare the abundance ratios of Pismis 18 with those presented in Magrini et al. (2017) and with the results of their chemical evolution model. The agreement is good for $\mathrm{Cr}, \mathrm{V}$ and $\mathrm{Ni}$, while the model predictions for $[\mathrm{Sc} / \mathrm{Fe}]$ are not able to explain the observational results.

\subsection{Heavy elements}

The slow- $\left(s^{-}\right)$and rapid- $(r-)$ neutron-capture elements are slightly enhanced, with $[\mathrm{Y} \mathrm{II} / \mathrm{Fe}]=0.06 \pm 0.10$, $[\mathrm{Zr} \mathrm{II} / \mathrm{Fe}]=0.20 \pm 0.07, \quad[\mathrm{Ba} \mathrm{II} / \mathrm{Fe}]=0.08 \pm 0.12, \quad[\mathrm{La} \mathrm{II} / \mathrm{Fe}]=$ $0.09 \pm 0.13$, and $[\mathrm{Ce} \mathrm{II} / \mathrm{Fe}]=0.07 \pm 0.10$ for the $s$-process elements and $[\mathrm{Nd} \mathrm{II} / \mathrm{Fe}]=0.09 \pm 0.13$ and $[\mathrm{Eu} \mathrm{II} / \mathrm{Fe}]=0.11 \pm 0.10$ for the $r$-process elements. The abundances of neutron capture elements for Pismis 18 are also included in the Magrini et al. (2018; hereafter M18) study. The small differences between the results presented here and in M18 are related to the more strict selection of stars included to compute the average value by M18, in which stars with large errors on individual abundances were not considered. In addition, for Eu, M18 adopted the solar value from Grevesse et al. (2007). An increase of the abundance of slow neutron capture elements is expected in the youngest stellar populations (D'Orazi et al. 2009; Maiorca et al. 2012; 


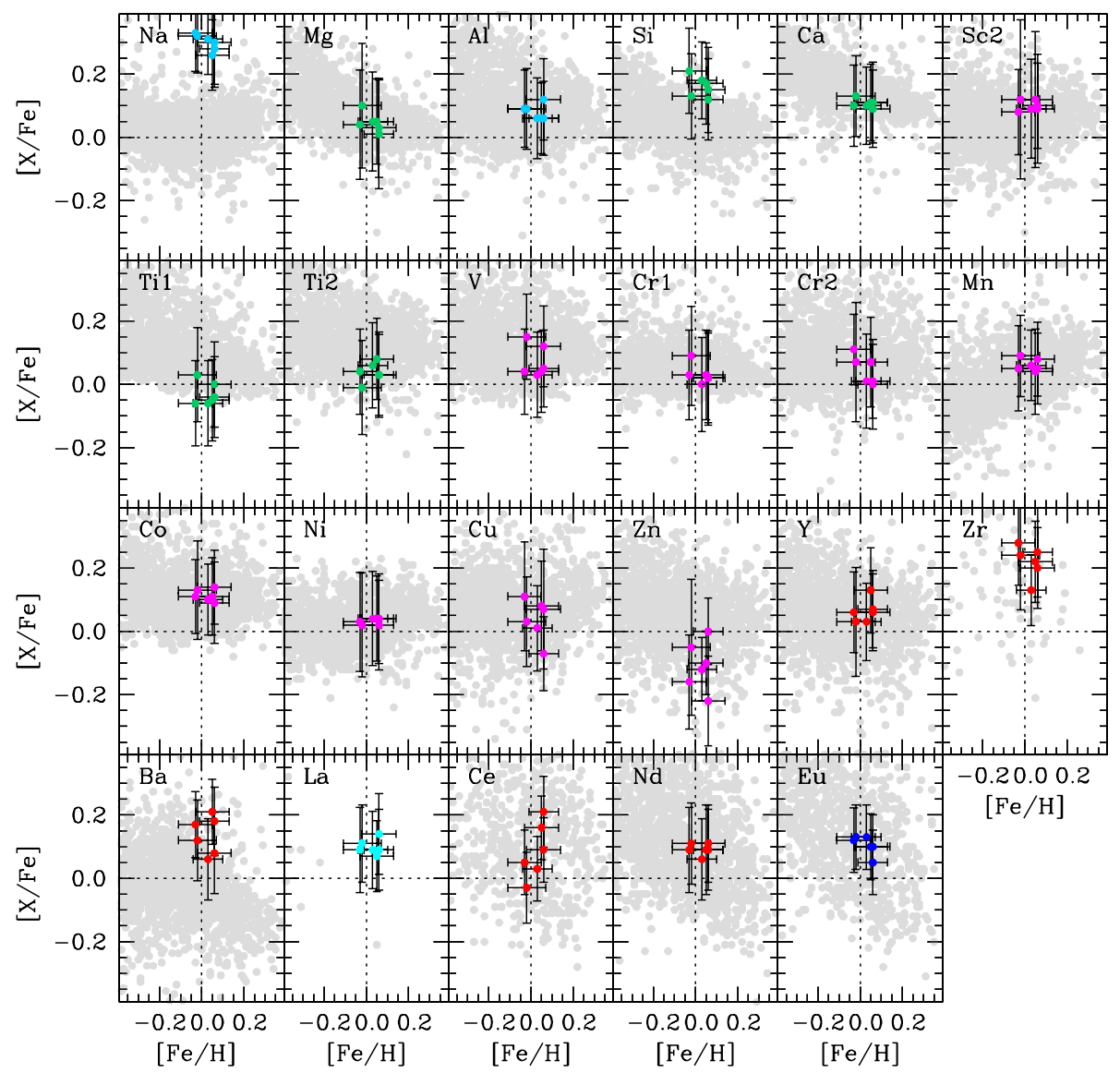

Fig. 9. Distribution of $[\mathrm{X} / \mathrm{Fe}]$ ratios as a function of $[\mathrm{Fe} / \mathrm{H}]$ for 23 species including light (cyan), $\alpha$ (green), Fe-peak (magenta) and $\mathrm{n}$-capture (red for $s$-process and blue for $r$ process) elements. The grey points correspond to field MW stars in GES (see text; it is noted that no $\mathrm{Y}$ measurements are available for them).
Spina et al. 2017) due to the strong contribution of low mass Asymptotic Giant Branch (AGB) stars, which, given their long lifetimes, restore their material to the interstellar medium at late times and hence can be incorporated only in the youngest generation of new born stars. In M18, we have studied the effect of the use $\mathrm{Fe}_{\text {II }}$ instead of $\mathrm{Fe}_{\mathrm{I}}$ to compute the abundances for elements with singly ionized atoms, which, in principle, would be more appropriate. However, there is a remarkable agreement between $\log (\mathrm{Fe} \mathrm{I} / \mathrm{H})$ and $\log (\mathrm{Fe} \mathrm{I} / \mathrm{H})$ in GES idR 5 samples. Thus, since the Fe II abundances are affected by larger uncertainties, we adopted in this paper the Fe I abundances to compute the $[\mathrm{X} / \mathrm{Fe}]$ abundance ratios.

As stated in the Introduction, OCs are considered to be simple stellar populations. This implies that all stars in the cluster share the same initial chemical composition and that any differences found should be attributed solely to evolutionary effects (e.g. diffusion, mixing) or to the presence of a companion. This homogeneity in chemical composition prompted the suggestion of chemical tagging, that is, of identifying the common origin of apparently unrelated stars (see e.g. the review by Freeman \& Bland-Hawthorn 2002). While no dedicated study, such as the one by Ness et al. (2018) on several APOGEE open clusters, has been performed for the GES cluster sample yet, we have not found any compelling indication of unexplained intrinsic spreads (see e.g. Cantat-Gaudin et al. 2014; Donati et al. 2014; Overbeek et al. 2017, just to cite M11, Trumpler 20, and Trumpler 23). The case of Pismis 18, although based only on six stars, does not differ from this trend, as it shows significant homogeneity in all elemental abundances: the error on the mean abundance (see Col. 2 of Table 9) ranges from 0.01 dex for several elements (e.g. Al I, Si I, Ca I, Ti I, Fe I, Fe II), to 0.02-0.04 dex (e.g. for Ti II, Cr II, Ba II), and rarely is larger than 0.05 dex. The

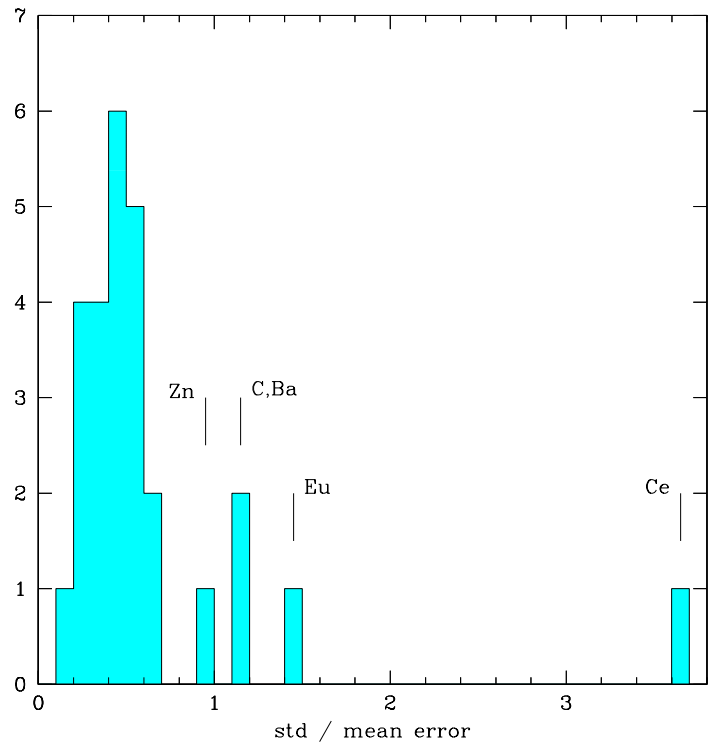

Fig. 10. Histogram of the ratio between average error and standard dispersion for all species ( $\mathrm{Li}$ excluded) measured for the high probability members of Pismis 18.

dispersion slightly increases when introducing the conversion to elemental ratios with respect to $\mathrm{H}$ and $\mathrm{Fe}$ (Cols. 3 and 4 of Table 9). As shown in Fig. 9, none of the 23 species shows any significant dispersion (see the error bars, indicating the average error on each star). As a further check, we plot in Fig. 10 the ratio of the rms of the $\log [\mathrm{X} / \mathrm{H}]$ values and the average error, using the values in Tables 4-6 for the calculations. In almost all cases the dispersion is smaller than the error, indicating homogeneity 
in composition. A few discrepant cases are indicated, with ratios larger than 1. Generally, this occurs for elements difficult to measure (e.g. Ba, with very strong lines, or $\mathrm{Ce}$, with only a few weak lines).

Figure 9 also shows the distribution of elemental ratios as a function of iron abundance for field MW stars observed by GES, for homogeneous comparison. We selected only stars observed with UVES (setup U580) and in the metallicity range -0.4 to 0.4; there are about 1700 stars, almost all dwarfs (the giants are about 20 in total). This paucity of giants explains the large difference $(>0.2 \mathrm{dex})$ we see for $[\mathrm{Na} / \mathrm{Fe}]$; in fact, giants of mass about $2-2.5 M_{\odot}$, such as those in Pismis 18, are expected to show enhanced $\mathrm{Na}$ abundance with respect to MS stars, due to mixing (see e.g. Smiljanic et al. 2016, 2018b for a discussion). For all other cases the distributions of cluster values fall within the range of field values.

\section{Summary and conclusions}

We have conducted an extended radial velocity and proper motion membership study as well as spectroscopic metallicity measurements for 142 stars in and around the inner disc Galactic cluster Pismis 18, using Gaia-ESO Survey iDR5 data, as well as Gaia DR2 data. Of the 142 stars, we could confirm high confidence membership for 26 stars, out of which six lie on the red clump and 20 on the upper MS of the cluster. These stars were used to determine the systemic velocity of the cluster, $-27.5 \pm 2.5 \mathrm{~km} \mathrm{~s}^{-1}$. Gaia DR2 photometry was used to re-determine cluster parameters based on high confidence member stars only. According to these new estimates, Pismis 18 has an age of $\tau=700_{-50}^{+40} \mathrm{Myr}$, interstellar reddening of $E(B-V)=0.562_{-0.026}^{+0.012} \mathrm{mag}$ and a de-reddened distance modulus $D M_{0}=11.96_{-0.24}^{+0.10}$ mas (corresponding to $2.47_{-0.26}^{+0.11} \mathrm{kpc}$ ). Using abundance measurements for 22 (i.e. not including $\mathrm{Li}$ ) elements based on high-resolution spectra of the six radial-velocity member stars on the red clump, we determined that the median metal abundance of Pismis 18 is above solar (using all measured elements) at $0.23 \pm 0.05 \mathrm{dex}$, with the ratio of the $\alpha$ elements to iron about solar (within the errors) at $[\alpha / \mathrm{Fe}]=0.07 \pm 0.13$. A slight enhancement was observed for neutron-capture elements, which is expected for younger disc populations.

Acknowledgements. Based on data products from observations made with ESO Telescopes at the La Silla Paranal Observatory under programme ID 188.B3002. These data products have been processed by the Cambridge Astronomy Survey Unit (CASU) at the Institute of Astronomy, University of Cambridge and by the FLAMES/UVES reduction team at INAF/Osservatorio Astrofisico di Arcetri. These data have been obtained from the Gaia-ESO Survey Data Archive, prepared and hosted by the Wide Field Astronomy Unit, Institute for Astronomy, University of Edinburgh, which is funded by the UK Science and Technology Facilities Council. This work was partly supported by the European Union FP7 programme through ERC grant number 320360 and by the Leverhulme Trust through grant RPG-2012-541. We acknowledge the support from INAF and Ministero dell' Istruzione, dell' Università' e della Ricerca (MIUR) in the form of the grant "Premiale VLT 2012". The results presented here benefit from discussions held during the Gaia-ESO workshops and conferences supported by the ESF (European Science Foundation) through the GREAT Research Network Programme. The research leading to these results has received funding from the European Community's Seventh Framework Programme (FP7-SPACE-2013-1) under grant agreement no. 606740. This work has made use of data from the European Space Agency (ESA) mission Gaia (https://www.cosmos.esa.int/gaia), processed by the Gaia Data Processing and Analysis Consortium (DPAC, https://www.cosmos.esa.int/ web/gaia/dpac/consortium). Funding for the DPAC has been provided by national institutions, in particular the institutions participating in the Gaia Multilateral Agreement. E. T. acknowledges the University of Pisa (Low and intermediate mass stellar models for the age determination of stellar clusters observed by the Gaia satellite, PI: S.Degl'Innocenti) and INFN (Iniziativa speci- fica TAsP). A. R. C. acknowledges support through the Australian Research Council through grant DP160100637. E. D. -M. acknowledges the support from Fundação para a Ciência e a Tecnologia (FCT) through national funds and from FEDER through COMPETE2020 by the following grants UID/FIS/04434/2013 \& POCI-01-0145-FEDER-007672, PTDC/FIS-AST/7073/2014 \& POCI-010145-FEDER-016880 and by the Investigador FCT contract IF/00849/2015. S. F. was supported by the project grant "The New Milky Way" from Knut and Alice Wallenberg Foundation. R. S. acknowledges support from the Polish Ministry of Science and Higher Education. A. B. acknowledges PREMIALE 2015 MITiC (PI B. Garilli).

\section{References}

Asplund, M., Grevesse, N., Sauval, A. J., \& Scott, P. 2009, ARA\&A, 47, 481 Bertelli Motta, C., Salaris, M., Pasquali, A., \& Grebel, E. K. 2017, MNRAS, 466, 2161

Bonatto, C., Bica, E., \& Girardi, L. 2004, A\&A, 415, 571

Bragaglia, A. 2018, in Astrometry and Astrophysics in the Gaia Sky, eds. A. Recio-Blanco, P. de Laverny, A. G. A. Brown, \& T. Prusti, IAU Symp., 330, 119

Bravi, L., Zari, E., Sacco, G. G., et al. 2018, A\&A, 615, A37

Cantat-Gaudin, T., Vallenari, A., Zaggia, S., et al. 2014, A\&A, 569, A17 Cantat-Gaudin, T., Donati, P., Vallenari, A., et al. 2016, A\&A, 588, A120 Cantat-Gaudin, T., Jordi, C., Vallenari, A., et al. 2018a, A\&A, 618, A93 Cantat-Gaudin, T., Vallenari, A., Sordo, R., et al. 2018b, A\&A, 615, A49 Carlberg, J. K. 2014, AJ, 147, 138

Casagrande, L. 2007, in From Stars to Galaxies: Building the Pieces to Build Up the Universe, eds. A. Vallenari, R. Tantalo, L. Portinari, \& A. Moretti, ASP Conf. Ser., 374, 71

Casamiquela, L., Carrera, R., Blanco-Cuaresma, S., et al. 2017, MNRAS, 470, 4363

Casamiquela, L., Carrera, R., Balaguer-Núñez, L., et al. 2018, A\&A, 610, A66 Chiappini, C. 2005, in Interacting Binaries: Accretion, Evolution, and Outcomes, eds. L. Burderi, L. A. Antonelli, F. D'Antona, et al., AIP Conf. Ser., 797, 476

Chiappini, C., Anders, F., Rodrigues, T. S., et al. 2015, A\&A, 576, L12

Cyburt, R. H. 2004, Phys. Rev. D, 70, 023505

Degl'Innocenti, S., Prada Moroni, P. G., Marconi, M., \& Ruoppo, A. 2008, Ap\&SS, 316, 25

Dell'Omodarme, M., Valle, G., Degl'Innocenti, S., \& Prada Moroni, P. G. 2012, A\&A, 540, A26

Dias, W. S., Alessi, B. S., Moitinho, A., \& Lépine, J. R. D. 2002, A\&A, 389, 871

Donati, P., Cantat Gaudin, T., Bragaglia, A., et al. 2014, A\&A, 561, A94

Donati, P., Bragaglia, A., Carretta, E., et al. 2015, MNRAS, 453, 4185

D’Orazi, V., Magrini, L., Randich, S., et al. 2009, ApJ, 693, L31

Drew, J. E., Gonzalez-Solares, E., Greimel, R., et al. 2014, MNRAS, 440, 2036 Freeman, K., \& Bland-Hawthorn, J. 2002, ARA\&A, 40, 487

Friel, E. D. 1995, ARA\&A, 33, 381

Friel, E. D., Donati, P., Bragaglia, A., et al. 2014, A\&A, 563, A117

Gaia Collaboration (Brown, A. G. A., et al.) 2016, A\&A, 595, A2

Gaia Collaboration (van Leeuwen, F., et al.) 2017, A\&A, 601, A19

Gaia Collaboration (Babusiaux, C., et al.) 2018a, A\&A, 616, A10

Gaia Collaboration (Brown, A. G. A., et al.) 2018b, A\&A, 616, A1

Gaia Collaboration (Helmi, A., et al.) 2018c, A\&A, 616, A12

Gaia Collaboration (Katz, D., et al.) 2018d, A\&A, 616, A11

Gilmore, G., Randich, S., Asplund, M., et al. 2012, The Messenger, 147, 25

Grevesse, N., Asplund, M., \& Sauval, A. J. 2007, Space Sci. Rev., 130, 105

Heiter, U., Lind, K., Asplund, M., et al. 2015, Phys. Scr., 90, 054010

Jackson, R. J., Jeffries, R. D., Lewis, J., et al. 2015, A\&A, 580, A75

Jacobson, H. R., Friel, E. D., Jílková, L., et al. 2016, A\&A, 591, A37

Jofré, P., Heiter, U., Worley, C. C., et al. 2017, A\&A, 601, A38

Kharchenko, N. V., Piskunov, A. E., Schilbach, E., Röser, S., \& Scholz, R.-D. 2013, A\&A, 558, A53

Krone-Martins, A., \& Moitinho, A. 2014, A\&A, 561, A57

Kruijssen, J. M. D., Pelupessy, F. I., Lamers, H. J. G. L. M., Portegies Zwart, S. F., \& Icke, V. 2011, MNRAS, 414, 1339

Kubryk, M., Prantzos, N., \& Athanassoula, E. 2013, MNRAS, 436, 1479

Lindegren, L., Hernández, J., Bombrun, A., et al. 2018, A\&A, 616, A2

Luri, X., Brown, A. G. A., Sarro, L. M., et al. 2018, A\&A, 616, A9

Magrini, L., Sestito, P., Randich, S., \& Galli, D. 2009, A\&A, 494, 95

Magrini, L., Randich, S., Donati, P., et al. 2015, A\&A, 580, A85

Magrini, L., Randich, S., Kordopatis, G., et al. 2017, A\&A, 603, A2

Magrini, L., Spina, L., Randich, S., et al. 2018, A\&A, 617, A106

Maiorca, E., Magrini, L., Busso, M., et al. 2012, ApJ, 747, 53

Malkin, Z. 2013, in Advancing the Physics of Cosmic Distances, ed. R. de Grijs, IAU Symp., 289, 406 
Marrese, P. M., Marinoni, S., Fabrizio, M., \& Altavilla, G. 2019, A\&A, 621, A144

Martig, M., Rix, H.-W., Silva Aguirre, V., et al. 2015, MNRAS, 451, 2230

Mermilliod, J.-C., Mayor, M., \& Udry, S. 2009, A\&A, 498, 949

Minchev, I., Chiappini, C., \& Martig, M. 2014, A\&A, 572, A92

Morales, E. F. E., Wyrowski, F., Schuller, F., \& Menten, K. M. 2013, A\&A, 560, A76

Ness, M., Rix, H.-W., Hogg, D. W., et al. 2018, ApJ, 853, 198

Netopil, M., Paunzen, E., Heiter, U., \& Soubiran, C. 2016, A\&A, 585, A150

Önehag, A., Gustafsson, B., \& Korn, A. 2014, A\&A, 562, A102

Overbeek, J. C., Friel, E. D., Donati, P., et al. 2017, A\&A, 598, A68

Pace, G., Pasquini, L., \& François, P. 2008, A\&A, 489, 403

Pancino, E., Lardo, C., Altavilla, G., et al. 2017, A\&A, 598, A5

Pasquini, L., Avila, G., Blecha, A., et al. 2002, The Messenger, 110, 1

Piatti, A. E., Clariá, J. J., Bica, E., Geisler, D., \& Minniti, D. 1998, AJ, 116 , 801

Portegies Zwart, S. F., Hut, P., Makino, J., \& McMillan, S. L. W. 1998, A\&A, 337,363

Randich, S., Sestito, P., Primas, F., Pallavicini, R., \& Pasquini, L. 2006, A\&A, 450,557

Randich, S., \& Gilmore, G., \& Gaia-ESO Consortium 2013, The Messenger, 154,47

Randich, S., Tognelli, E., Jackson, R., et al. 2018, A\&A, 612, A99

Riess, A. G., Casertano, S., Yuan, W., et al. 2018, ApJ, 861, 126
Robin, A. C., Reylé, C., Derrière, S., \& Picaud, S. 2003, A\&A, 409, 523 Sacco, G. G., Morbidelli, L., Franciosini, E., et al. 2014, A\&A, 565, A113 Sestito, P., Bragaglia, A., Randich, S., et al. 2008, A\&A, 488, 943 Shetrone, M. D., \& Sandquist, E. L. 2000, AJ, 120, 1913

Smiljanic, R., Korn, A. J., Bergemann, M., et al. 2014, A\&A, 570, A122

Smiljanic, R., Romano, D., Bragaglia, A., et al. 2016, A\&A, 589, A115

Smiljanic, R., Franciosini, E., Bragaglia, A., et al. 2018a, A\&A, 617, A4

Smiljanic, R., Donati, P., Bragaglia, A., Lemasle, B., \& Romano, D. 2018b, A\&A, 616, A112

Spina, L., Randich, S., Magrini, L., et al. 2017, A\&A, 601, A70

Stassun, K. G., \& Torres, G. 2018, ApJ, 862, 61

Tadross, A. L. 2008, New Astron., 13, 370

Tang, B., Geisler, D., Friel, E., et al. 2017, A\&A, 601, A56

Tautvaišiene, G., Edvardsson, B., Tuominen, I., \& Ilyin, I. 2000, A\&A, 360, 499

Tognelli, E., Prada Moroni, P. G., \& Degl'Innocenti, S. 2011, A\&A, 533, A109

Tognelli, E., Prada Moroni, P. G., \& Degl'Innocenti, S. 2018, MNRAS, 476, 27

Valle, G., Dell'Omodarme, M., Prada Moroni, P. G., \& Degl'Innocenti, S. 2017, A\&A, 600, A41

Yong, D., Carney, B. W., \& Teixera de Almeida, M. L. 2005, AJ, 130, 597

Yong, D., Carney, B. W., \& Friel, E. D. 2012, AJ, 144, 95

Zinn, J. C., Pinsonneault, M. H., Huber, D., \& Stello, D. 2018, ApJ, submitted [arXiv:1805.02650] 